\title{
SHAPE OPTIMIZATION IN LAMINATED COMPOSITE PLATES
}

\author{
Myung S. LEE* \\ Ford Scientific Research Laboratories, Dearhorn, MI, U.S.A. \\ Noboru KIKUCHI and Richard A. SCOTT \\ Department of Mechanical Engineering and Applied Mechanics, University of Michigan, \\ Ann Arbor, MI, U.S.A.
}

Received 12 July 1987

Revised manuscript received 5 July 1988

\section{Introduction}

At this stage a substantial body of literature exists for shape optimization involving isotropic materials. The reader is referred to the articles of Haug [1] and Haftka and Grandhi [2] for overviews of the material.

One approach to the problem is analytical. Calculus of variation techniques are employed to derive explicit optimality conditions. Choi and Haug [3], Dems and Mroz [4], and Banichuk $[5,6]$ have adopted this approach. Of note is the recent work of Kikuchi, Chung, Torigaki and Taylor [7] who used variational methods together with an adaptive grid scheme developed by Diaz, Kikuchi and Taylor [8] for shape optimization in linear elastic structures. The works of Braibant and Fleury $[9,10]$, involving B-splines, should also be cited.

Up to now, structural optimization of laminated composites has involved ply orientations and thicknesses as design variables. To the authors' knowledge, no work on shape optimization of laminated structures has been done. Kicher and Chao [11] considered the optimum design of fiber composite cylinders under combined axial, radial, and torsional loads. Minimum weight was taken as the objective function, with the ply thickness being the design variable. Hirano [12, 13], Park [14], and Tauchert and Adibhatla [15] treated the optimum design of laminated plates under various types of loading. The objective functions used were maximum buckling load, first ply failure (see [16]), and minimum strain energy, respectively. Hirano and Park used fiber angles as design variables, whereas Tauchert and Adibhatla employed both fiber angles and layer thickness as variables. Yiping [17] treated the optimal design of a laminated plate with several elliptical holes under uniaxial tensile loading. Minimizing the maximum value of the Tsai-Wu failure functional (see [18]) was the objective and the fiber angle was the design variable. In all of the above works on composites, mathematical programming techniques were used to obtain the solutions. Other approaches have been used. Bauchau [19] considered the optimal design of rotating graphite-epoxy shafts.

* This work forms part of a dissertation submitted in partial fulfilment of the requiremuits tor the degree of Doctor of Philosophy (Applied Mechanics) at the University of Michigan, 1987. 
The objective function involved the first natural bending frequency and ply thickness was used as the design variable. An optimality condition was derived and an iterative algorithm developed for the solution.

In view of the lack of information on shape optimization in composite media, it was decided to do a pilot study involving a hole in a laminated plate subject to in-plane biaxial tension. Classical plate lamination theory is used, together with a finite element approach. Minimizing the maximum values of certain failure functions $\Phi$ (Tsai-Hill, see [18], and Tsai-Wu) is taken to be the objective. In the single-ply case it can be shown that the optimality condition is that the mutual strain energy is constant on the design boundary under the condition that the design boundary has no geometrical constraint (see $[7$, eq. 10; 20]). For isotropic media, it has also been shown that the optimality condition is $\Phi=$ constant on the hole boundary. This, so far, has not been proven for anisotropic media. Here, analogous to the isuiropic case, it is postulated that the maximum value of $\Phi$ occurs on the hole boundary. At all stages of the numerical work this was monitored numerically and found to be true. Moreover $\Phi=$ constant on the hole boundary is postulated as the optimality condition. This, in general, will not lead to a global optimum (in view of the possibility of multiple holes), but it should lead to a local minimum.

Of particular interest was to see whether the techniques developed for shape optimization in isotropic media would work for laminated composites, involving as they do much larger stress gradients.

\section{Weak formulation}

The coordinate systems employed are shown in Fig. .. $x_{1}, x_{2}$ are global coordinates, whereas $x_{1}^{\prime}, x_{2}^{\prime}$ are the principal material coordinates in the fiber direction and perpendicular to the fiber direction, respectively.

For plane stress the equations of equilibrium are, in the absence of body forces,

$$
\sigma_{i j, j}=0 \text { in the domain } \Omega \text {. }
$$

Here, and throughout, standard index notation is used, with the range of the indices being from 1 to 2 , unless otherwise specified.

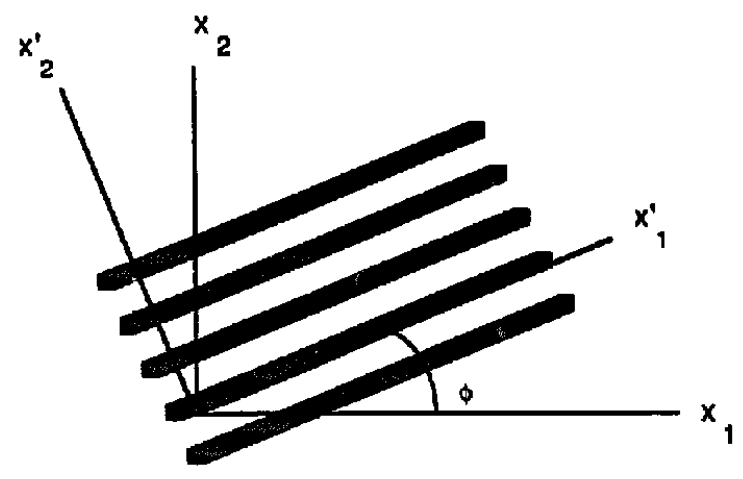

Fig. 1. Coordinate systems employed. 
Displacement boundary conditions are

$$
u_{i}=g_{i} \text { on the boundary } \Gamma_{u} \text {, }
$$

where $g_{i}$ are specified functions. Stress boundary conditions are

$$
\sigma_{i j} n_{j}=t_{i} \text { on the boundary } \Gamma_{\imath},
$$

where $t_{i}$ are components of the applied surface traction vector per unit thickness.

Multiplying (1) by arbitrary virtual displacements $\bar{v}_{v}$ (note $\bar{v}_{i}=0$ on $\Gamma_{u}$ ), summing, and integrating one obtains

$$
\int_{\Omega} \sigma_{i j, j} \bar{u}_{i} \mathrm{~d} \Omega=0,
$$

or, applying the divergence theorem and using the boundary conditions,

$$
\int_{\Omega} \sigma_{i j} \bar{v}_{i, j} \mathrm{~d} \Omega=\int_{r_{i}} t_{i} \bar{u}_{\imath} \mathrm{d} \Gamma .
$$

For a single lamina, the constitutive law is

$$
\sigma_{i j}=E_{i j k l} \varepsilon_{k l}=\frac{1}{2} E_{i j k l}\left(u_{k, l}+u_{l, k}\right),
$$

where the elastic moduli satisfy

$$
E_{i j k l}=E_{j i k l}=E_{k l i j} .
$$

Then (4) may be written

$$
\int_{\Omega} E_{i j k l} u_{i, j} \bar{v}_{k, l} \mathrm{~d} \Omega=\int_{\Gamma_{t}} t_{i} \bar{v}_{i} \mathrm{~d} \Gamma
$$

which constitutes the weak formulation of the problem. A reduced form of the stiffness matrix, $E_{m n}$, is customarily employed:

$$
\left[\begin{array}{lll}
E_{1111} & E_{1122} & E_{1112} \\
& E_{2222} & E_{2212} \\
S Y M & & E_{1212}
\end{array}\right]=\left[\begin{array}{ccc}
E_{11} & E_{12} & E_{13} \\
& E_{22} & E_{23} \\
S Y M & & E_{33}
\end{array}\right] .
$$

For the single lamina, $E_{m n}$ is the so-called trarsformed reduced stiffness matrix $\bar{Q}_{m n}^{p}$, the first term of which is

$$
\bar{Q}_{11}^{p}=Q_{11}^{p^{\prime}} \cos ^{4} \varphi+\left(2 Q_{12}^{p^{\prime}}+Q_{33}^{p^{\prime}}\right) \sin ^{2} \varphi \cos ^{2} \varphi+Q_{22}^{p^{\prime}} \sin ^{4} \varphi,
$$

where $Q_{m n}^{p^{\prime}}$ is the reduced stiffness matrix, which involves the various Young's moduli and Poisson's ratios. Details can be found in Jones [18]. 

by

For multiple layers, classical plate lamination theory leads to a set of averaged moduli given

$$
E_{m n}=\frac{1}{H} \sum_{p=1}^{N} h^{p} \bar{Q}_{m n}^{p},
$$

where $h^{p}$ is the thickness of the $p$ th lamina, and $H$ is the total thickness of the laminate.

\section{Finite elements}

The domain $\Omega$ is discretized into a total NE of finite elements $\boldsymbol{\Omega}_{e}$. Standard finite element methodology leads to

$$
u_{i \gamma}=g_{i \gamma} \text { on the discretized boundary } \Gamma_{u \gamma},
$$

where $\gamma$ ranges from one to the total number of boundary nodes on $\Gamma_{u}$, and

$$
\sum_{e=1}^{\mathrm{NE}} \sum_{i=1}^{2} \sum_{\alpha=1}^{\mathrm{ND}} E_{i \alpha j \beta}^{e} u_{i \alpha}=\sum_{e=1}^{\mathrm{NE}_{2}} f_{j \beta}^{e},
$$

where

$$
\begin{aligned}
& E_{i \alpha j \beta}^{\mathrm{c}}=\int_{\Omega_{e}} E_{i k j l} \frac{\partial N_{\alpha}}{\partial x_{k}} \frac{\partial N_{\beta}}{\partial x_{l}} \mathrm{~d} \Omega, \\
& f_{j \beta}^{\mathrm{e}}=\int_{T_{t e}} t_{j} N_{\beta} \mathrm{d} \Gamma .
\end{aligned}
$$

In the above, $\mathrm{ND}$ is the number of nodes in an element, $\mathrm{NE}_{2}$ is the number of line elements on the traction boundary, $N_{\alpha}, N_{\beta}$ stand for shape functions, and $\Gamma_{t e}$ denotes a boundary segment on which traction is applied.

Two types of shape functions will be studied later, namely isoparametric QUAD4 and QUAD9 elements. Before going into details, the shape optimization problem will first be discussed.

\section{Shape optimization definitions}

The shape optimization problem studied here is as follows. Consider a composite infinite plate with a central hole, boundary $\Gamma_{0}$, subject to a uniform biaxial tensile stress applied at infinity. It is postulated that maximum strength is achieved by minimizing the maximum value of some appropriate failure function. The issue then is: what shape of the hole minimizes the maximum value of the failure function? Lamina (ply) failure and laminate failure must be treated separately. 
Anisotropic ply failure functions are extensions of isotropic yield criteria. Various anisotropic failure theories are present in the literature [18]. Typically they involve polynomial functions $\Phi$ of the stress components. Failure occurs when $\Phi=1$. Two popular failure criteria for anisotropic plies, the Tsai-Hill and Tsai-Wu failure criteria, are chosen for study since experimental data [18] show reasonable agreement with them.

The Tsai-Hill failure function is

$$
\Phi=\left(\frac{\sigma_{x i} x_{i}^{i}}{X}\right)^{2}-\frac{\sigma_{x_{1}^{i} x_{i}^{\prime}} \sigma_{x_{2}^{i} x_{i}^{\prime}}}{X^{2}}+\left(\frac{\sigma_{x_{2} x_{2}^{\prime}}}{Y}\right)^{2}+\left(\frac{\sigma_{x_{1}^{i} x_{i}^{\prime}}}{S}\right)^{2},
$$

where $X$ and $Y$ are the uniaxial tensile strengths parallel and perpendicular to the fibers, and $S$ is the lamina shear strength. The Tsai-Wu function is

where

$$
\begin{aligned}
\Phi= & F_{x_{1}^{\prime} x_{1}} \sigma_{x_{1}^{\prime} x_{i}^{\prime}}^{2}+2 F_{x_{1}^{\prime} x_{2}^{\prime}} \sigma_{x_{1}^{\prime} x_{1}^{\prime}} \sigma_{x_{2}^{\prime} x_{2}^{\prime}}+F_{x_{2}^{\prime} x_{2}^{\prime}} \sigma_{x_{2}^{\prime} x_{2}^{\prime}}^{2} \\
& +F_{s s} \sigma_{x_{1}^{\prime} x_{2}^{\prime}}^{2}+F_{x_{1}^{\prime}} \sigma_{x_{1}^{\prime} x_{i}^{\prime}}+F_{x_{2}^{\prime}} \sigma_{x_{2}^{\prime} x_{2}^{\prime}},
\end{aligned}
$$

$$
\begin{aligned}
& F_{x_{1}^{\prime} x_{1}^{\prime}}=\frac{1}{X_{\mathrm{t}} X_{\mathrm{c}}}, \quad F_{x_{2}^{\prime} x_{2}^{\prime}}=\frac{1}{Y_{\mathrm{t}} Y_{\mathrm{c}}}, \quad F_{s s}=\frac{1}{S^{2}}, \\
& F_{x_{1}^{\prime} x_{2}^{\prime}}=\frac{1}{2 \sigma^{2}}\left(1-\left\{\frac{1}{X_{\mathrm{t}}}-\frac{1}{X_{\mathrm{c}}}+\frac{1}{Y_{\mathrm{t}}}-\frac{1}{Y_{\mathrm{c}}}\right\} \sigma-\left\{\frac{1}{X_{\mathrm{t}} X_{\mathrm{c}}}+\frac{1}{Y_{\mathrm{t}} Y_{\mathrm{c}}}\right\} \sigma^{2}\right), \\
& F_{x_{1}^{\prime}}=\frac{1}{X_{\mathrm{t}}}-\frac{1}{X_{\mathrm{c}}}, \quad F_{x_{2}^{\prime}}=\frac{1}{Y_{\mathrm{t}}}-\frac{1}{Y_{\mathrm{c}}} .
\end{aligned}
$$

$X_{\mathrm{t}}, Y_{\mathrm{t}}$ and $X_{\mathrm{c}}, Y_{\mathrm{c}}$ are the lamina longitudinal and transverse strengths in tension and compression, respectively, and $\sigma$ is the biaxid tensile failure stress.

To avoid unrealistic designs, a constraint in the form of a bound on the area must be specified. Here the area of the hole is taken to be constant. For plates of finite size (which are the ones studied numerically), this constraint is equivalent to

$$
\int_{\Omega} \mathrm{d} \Omega-\bar{A}=\mathbf{0},
$$

where $\bar{A}$ is the original area of $\Omega$. The optimization problem for laminae can then be stated:

$$
\operatorname{minimize} \Phi_{0}=\min _{\Gamma_{0}} \max _{\left(x_{1}, x_{2}\right) \in \bar{\Omega}} \Phi,
$$

subject to the isoperimetric constraint (19), where $\bar{\Omega}$ is the closure of $\Omega$.

For the isotropic case, $\Phi$ is usually taken as the Von Mises function. Using the properties of harmonic functions (stress potentials) and the maximum principle, Banichuk [5] proved that the maximum value of $\Phi$ occurred on the hole boundary. Moreover, he proved that the optimality condition of the problem given by $(20)$ is that $\Phi$ is a constant on the hole boundary. In the anisotropic and the composite laminate cases, potential functions do not, in general, exist and to the authors' knowledge no one has proved that the maximum value of the failure function occurs on the boundary or that the optimality condition leads to $\Phi=$ constant on the 
boundary. An operational procedure is adopted here as follows: At every step of the calculations, the location of the maximum value of $\Phi$ is determined. In all cases studied, it was found to be on the hole boundary. Moreover, the condition

$$
\Phi=\text { constant on } \Gamma_{0}
$$

is postulated as the optimality condition.

Laminate failure poses further problems. Failure of composite laminates are complicated by a multitude of independent and interacting mechanisms which include filament breakings, delamination, and crack propagation, etc. There are two failure criteria frequently used for composite laminates. One is the first ply failure (FPF) criterion, described by Tsai and Hahn [16] in which failure in one particular ply is regarded as total laminate failure. The other is the last ply failure (LPF) criterion, adopted by Soni [21], in which failure in last ply is regarded as total laminate failure.

If first ply failure is adopted as a criterion, the optimization problem can be stated by

$$
\left(\operatorname{Min}_{\Gamma_{0}} \underset{i=1, \ldots, N}{\operatorname{Max}} \underset{\left(x_{1}, x_{2}\right) \in \bar{\Omega}}{\operatorname{Max}} \Phi_{l}\right),
$$

where $N$ is the number of plies and $\Phi_{l}$ denotes the failure function in the Ith ply. Attempts to use (22) were not successful. It led to large, nonconvergent oscillations in the design boundary, and so FPF was not pursued.

If last ply failure is taken as a criterion, an optimization problem may be posed as follows:

$$
\operatorname{Min}\left(\underset{I=1, \ldots, N}{\operatorname{Min}} \operatorname{Max}_{\left(x_{1}, x_{2}\right) \in \delta} \Phi_{l}\right) \text {. }
$$

However, in using LPF, the stiffness matrix has to be continuously updated. A ply, once failed, is regarded as having zero stiffness. The formulation (23) does not take this into account. Alternate formulations were not evident and so LPF was not pursued either.

In the present study on composite laminates an average failure criterion is hypothesized. The avcrage failure functions, $\Phi_{\text {ave }}$, are obtained by averaging the failure function values through the plies and the total laminate is regarded as failing when the maximum value of the average failure function reaches 1 . Then (20) is replaced by

$$
\text { minimize } \Phi_{0}=\min _{\Gamma_{0}} \max _{x_{1}, x_{2} \in \bar{\Omega}} \Phi_{\text {ave }} .
$$

Again we postulate that the maximum value occurs on the hole boundary (checked at each step numerically) and that optimality is achieved by $\Phi_{\text {ave }}=$ constant on the hole boundary.

\section{Choice of element type}

For isotropic materials, a substantial body of experience has been accumulated on the type of finite element best suited to a given problem. For a great many cases that element has been 
found to be the eight-node or nine-node quadrilateral isoparametric element. For smooth enough solutions higher-order finite elements provide more accurate solutions. However, in anisotropic media, stress gradients can be substantially larger than in isotropic materials and so choices based on that experience cannot automatically be made. Some pilot studies were done to assess the relative merits of QUAD4 versus QUAD9 isoparametric elements. In particular studies were made on the stresses in both isotropic and anisotropic plates with a central hole under uniaxial tension (for which analytic results are available in the literature). Three regular uniform meshes as shown in Fig. 2 are used for the convergence test of QUAD4 and QUAD9 elements. In order to relate the number of divisions to the size of element for

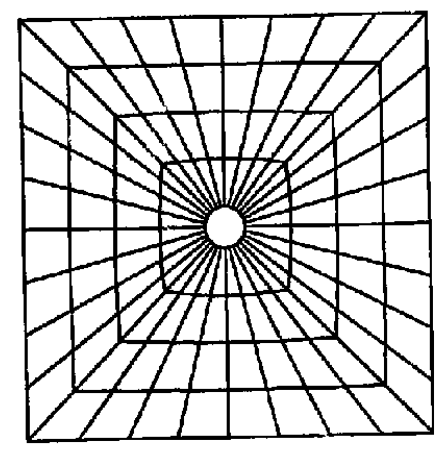

(I) $\mathrm{NE}=128 ; \mathrm{NX}=160$

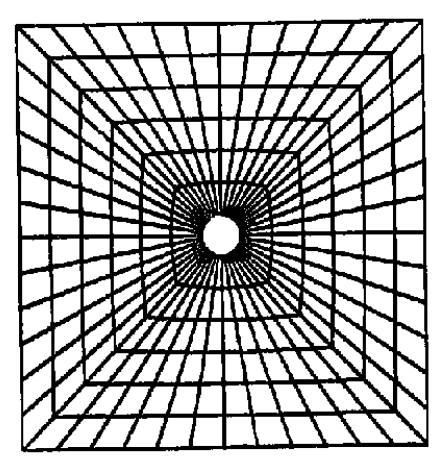

(I) $\mathrm{NE}=288 ; \mathrm{NX}=336$

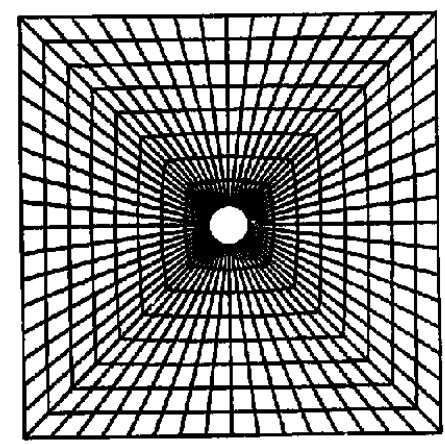

(III) $\mathrm{NE}=512 ; \mathrm{NX}=576$

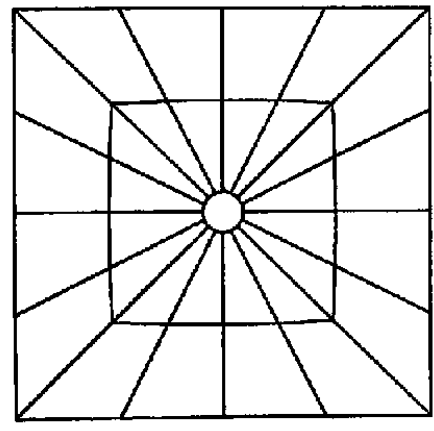

(I) $\mathrm{NE}=32 ; \mathrm{NX}=160$

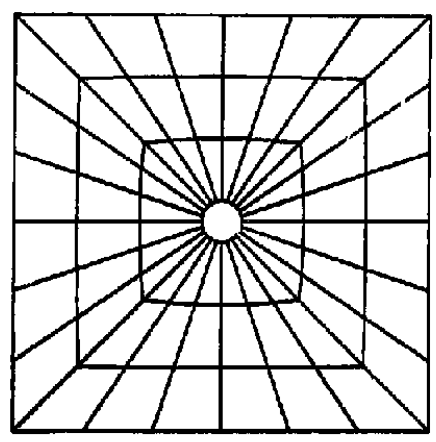

(II) $\mathrm{NE}=72 ; \mathrm{NX}=\mathbf{3 3 6}$

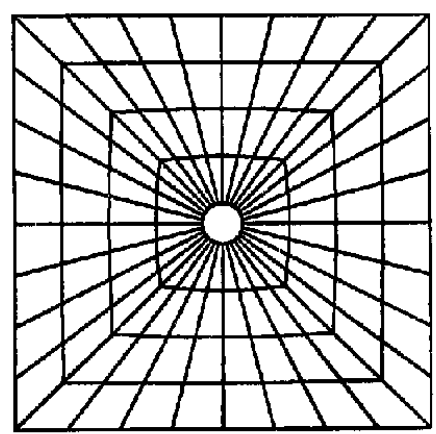

(III) $\mathrm{NE}=128 ; \mathrm{NX}=576$

Fig. 2. Meshes used. NE is total number of elements. NX is total number of nodes. 
convergence analysis, one QUAD9 element is arranged to be four QUAD4 elements, i.e., the mid-nodes of the QUAD9 elements are located exactly at the centers of edges. The number of divisions in both the $r$ - and $\theta$-directions are uniformly increased by the same amount.

The item of comparison was taken to be the maximum value of $\left|\sigma_{\theta \theta}-\sigma_{\theta \theta h}\right|$ on the hole boundary. Here the circumferential stresses $\sigma_{\theta \theta}$ and $\sigma_{\theta \theta h}$ represent the analytic solution and the numerical solution by the finite element method, respectively. The dimensions are such that infinite plate solutions can be used. For the isotropic case, the dimensional recommendations of Krauthammer [22] have been followed. For the anisotropic case, the dimensions are based on the work of Nuismer and Whitney [23] who used analytic results from Lekhnitskii's book [24]. Dimensions satisfying both cases are

$$
L / W=1 ., \quad L / D=10 . \quad(L=W=40 \mathrm{~cm}, \quad D=4 \mathrm{~cm}) .
$$

(The plate thickness is taken to be $1 \mathrm{~cm}$.) $L$ and $W$ represent length and width of the plate, respectively. $D$ is the diameter of the centra! hole.

The analytic solution for $\sigma_{\theta \theta}$ on the hole boundary for anisotropic plates under uniform tension was given by Lekhnitskii [24]. The Lekhnitskii solution is

$$
\begin{aligned}
\sigma_{\theta \theta}=P \frac{E_{\theta \theta}}{E_{x j x_{i}}}\{ & \left(-\cos ^{2} \varphi+(k+n) \sin ^{2} \varphi\right) k \cos ^{2} \theta \\
& +\left((1+n) \cos ^{2} \varphi-k \sin ^{2} \varphi\right) \sin ^{2} \theta \\
& -n(1+k+n) \sin \varphi \cos \varphi \sin \theta \cos \theta\},
\end{aligned}
$$

where $P$ is tensile stress applied at infinity in the $x_{1}$-direction, $\varphi$ is the angle measured from $x_{1}$-axis to $x_{1}^{\prime}$-axis (in ccw direction),

$$
\begin{aligned}
& k=\left(E_{x i x_{1}^{\prime}} / E_{x_{2}^{\prime} x_{2}^{\prime}}\right)^{1 / 2}, \\
& n=\left\{2\left(k-\nu_{x_{i}^{\prime} x_{2}^{\prime}}\right)+\left(E_{x j x_{1}^{\prime}} / G_{x_{i}^{\prime} x_{2}^{\prime}}\right)\right\}^{1 / 2},
\end{aligned}
$$

$G_{x_{1}^{\prime} x_{2}^{\prime}}$ is the shear modulus measured in the laminate coordinates, $\nu_{x_{1}^{\prime} x_{2}^{\prime}}$ is the major Poisson's ratio measured in the laminate coordinates, $E_{x x_{i}^{\prime}}$ is Young's modulus in the fiber direction, $E_{x_{2}^{\prime} x_{2}^{\prime}}$ is Young's modulus in the direction perpendicular to the fiber, and

$$
\frac{1}{E_{\theta \theta}}=\frac{\sin ^{4} \theta}{E_{x x_{i}^{\prime}}}+\left(\frac{1}{G_{x x_{i}^{\prime}}}-\frac{2 \nu_{x i x_{i}^{\prime}}}{E_{x x_{i}^{\prime}}}\right) \sin ^{2} \theta \cos ^{2} \theta+\frac{\cos ^{4} \theta}{E_{x_{2}^{\prime} x_{2}^{\prime}}} .
$$

Note that in Lekhnitskii's book ([24, eq. (39.1), p. 171]) the square root in (26) is left out. This typographical error was pointed out by Hyer [25].

Figure 3 shows graphs calculated from the exact solution (25) of the distribution of $\sigma_{\theta \theta}$ around the hole boundary. The anisotropic materials used are glass/epoxy and graphite/epoxy with $45^{\circ}$ fiber angles. The material properties are given in Table 1. For the isotropic material, Young's modulus $E=210 \mathrm{Gpa}$ and Poisson's ratio $\nu=0.29$. In the figure, the outward and inward arrows denote tension and compression, respectively. It is seen that in anisotropic 


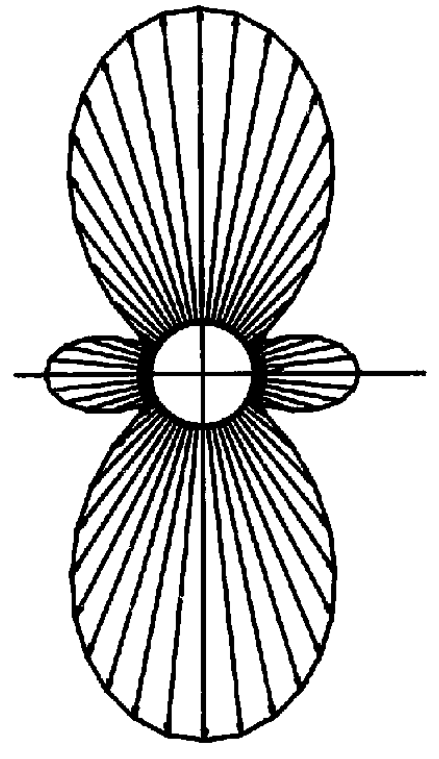

Isotropic

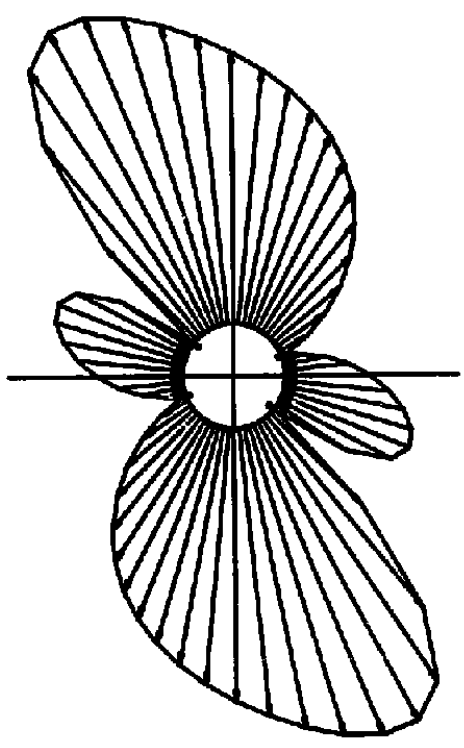

Glass/Dpoxy

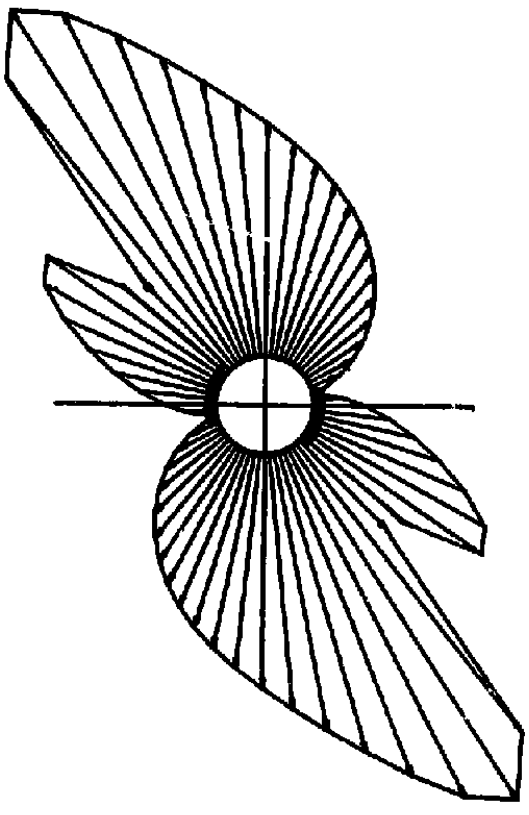

Graphite/Epoxy

Fig. 3. Distribution of $\sigma_{\theta \theta}$ on the hole boundary.

Table 1

Material properties of GL/EP and GR/EP

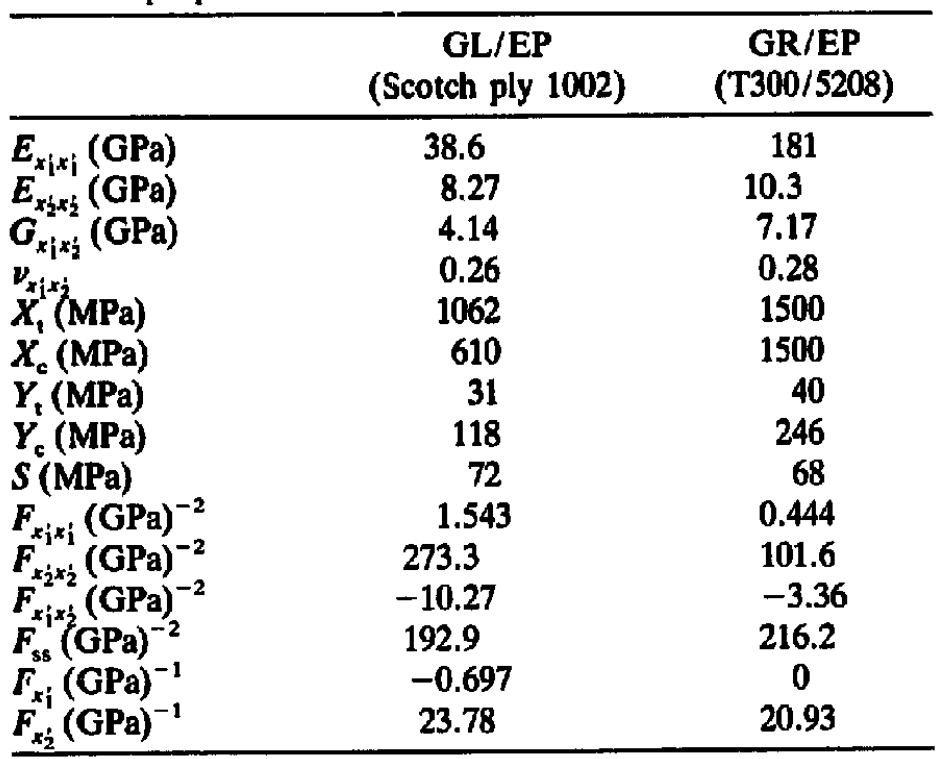

plates, the circumferential gradients of the circumferential stress are very large compared to these in the isotropic plate. For example, the maximum gradient in glass/epoxy is 2.5 times that in the isotropic case. For graphite/epoxy the maximum gradient is 7.5 times higher. From this, it is to be expected that greater difficulties will be encountered in analyzing anisotropic plates using numerical methods.

In the finite element work with QUAD4 and QUAD9 elements, the materials studied were 
steel and glass/epoxy (with a $45^{\circ}$ fiber angle). The meshes employed are as in Fig. 2. Note that uniform mesh refinements are used (which is the process used in mathematical studies of convergence). For both elements, the stresses computed are Cartesian components evaluated at the Gaussian integration points. These stresses are extrapolated to the nodal points using a global, least-squares smoothing procedure (see, e.g., [26]) and then the stress transformation laws yield the circumferential stress $\sigma_{\theta \theta h}$ (note that the reverse procedure is less accurate).

Figure 4 shows results for an applied axial stress of $100 \mathrm{~N} / \mathrm{cm}^{2}$, which leads to exact maximum values of $\sigma_{\theta \theta}=300 \mathrm{~N} / \mathrm{cm}^{2}$ and $\sigma_{\theta \theta}=325.40 \mathrm{~N} / \mathrm{cm}^{2}$ for the isotropic and GL/EP cases, respectively. Shown are convergence rates for the two elements together with the tabular data from which they are generated. Several points can be made. It can be shown mathematically that the theoretical convergence rate for the QUAD9 should be twice that for the QUAD4 for sufficiently smooth solutions. This behavior is not seen here, for the current level of remeshing. In fact it is seen that the convergence rates are very similar. An important point is that uniform meshing with both types of element leads to unacceptable inaccuracies in the stresses. Convergence tests were also performed using irregular meshes. Comparable, poor performance for both QUAD4 and QUAD9 was found (see [27] for details).

To gain some insight as to why the poor performance, $\sigma_{\theta \theta h}$ and $\sigma_{r r h}$, along a line at $45^{\circ}$ to the $x_{1}$-axis, as obtained from the global smoothing procedure (a least-squares method) are compared with the raw values obtained from a set of adjacent Gaussian integration points. For
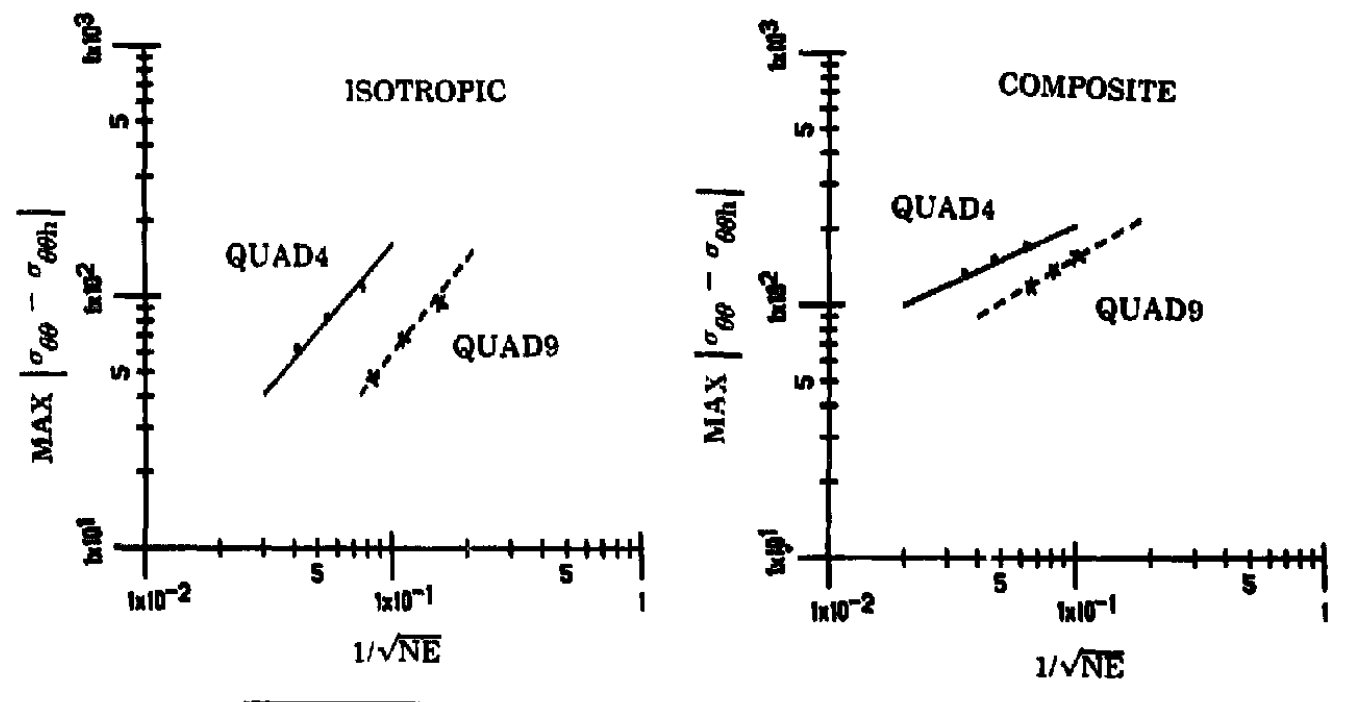

\begin{tabular}{cccccc}
\hline & \multicolumn{2}{c}{ Isotropic } & & \multicolumn{2}{c}{ Composite (GL/EP 45 ) } \\
\cline { 2 - 3 } \cline { 5 - 6 } Mesh type & max $\left|\sigma_{\theta \theta}-\sigma_{\theta \theta h}\right|^{\prime \prime}$ & & \multicolumn{2}{c}{$\max \left|\sigma_{\theta \theta}-\sigma_{\theta \theta h}\right|^{\mathrm{b}}$} \\
\cline { 2 - 3 } \cline { 5 - 6 } & QUAD4 & QUAD9 & & QUAD4 & QUAD9 \\
\hline I & 113.50 & 101.70 & & 177.50 & 171.30 \\
III & 85.60 & 71.80 & & 164.90 & 158.90 \\
III & 64.60 & 50.20 & & 148.50 & 142.40 \\
\hline
\end{tabular}

"Exact solution for isotropic case: $\max \sigma_{\theta \theta}=300.00$ (unit: $100 \mathrm{~N} / \mathrm{cm}^{2}$ ).

bxact solution for composite case: $\max \sigma_{\theta \theta}=325.40$ (unit: $100 \mathrm{~N} / \mathrm{cm}^{2}$ ).

Fig. 4. A comparison of convergence rates and errors for QUAD4 and QUAD9 regular meshes. 
a type-III mesh the results are shown in Figs. 5 and 6, for a GL/EP plate. Far away from the hole, both procedures give the same stress values. However, as the hole boundary is approached, the raw stresses obtained at the Gaussian integration points take on an erratic, jumpy behavior (because of the large stress gradients arising near the hole boundary). The poor quality of these stresses leads in turn to poor quality in the smoothed stresses, which suggests that fine meshes rather than uniform meshes should be used near the hole boundary. The major lesson learned from the overall study is that some adaptive mesh scheme should be used, instead of uniform meshing.

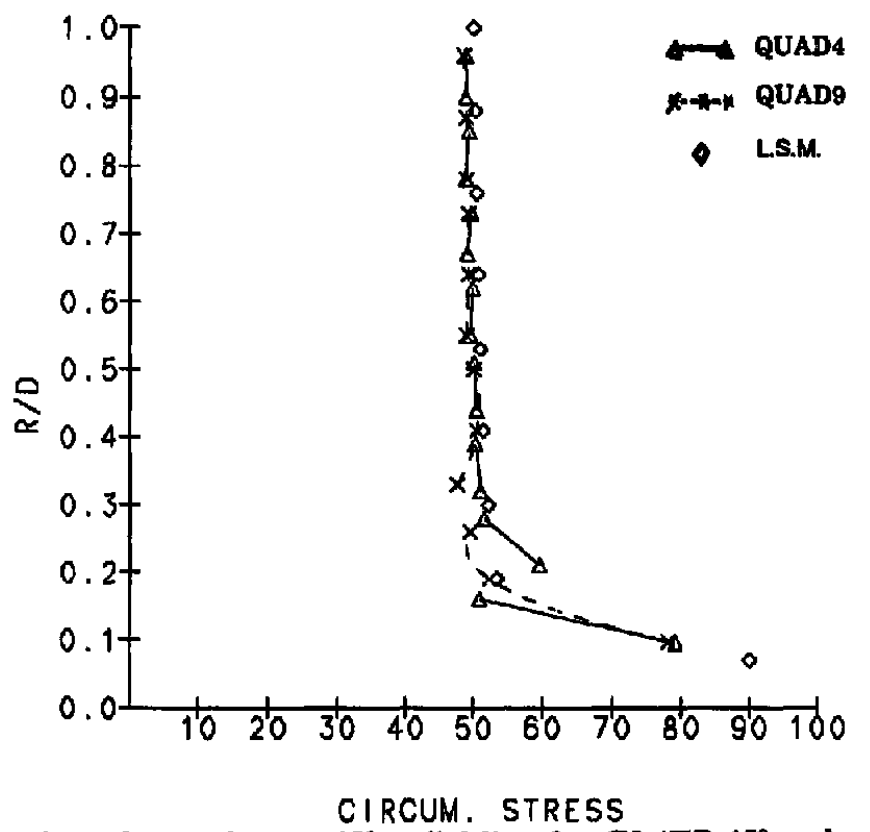

Fig. 5. Distribution of $\sigma_{\theta \theta h}$ along a $45^{\circ}$ radial line for $\mathrm{GL} / \mathrm{EP} 45^{\circ}$ and a type-III mesh.

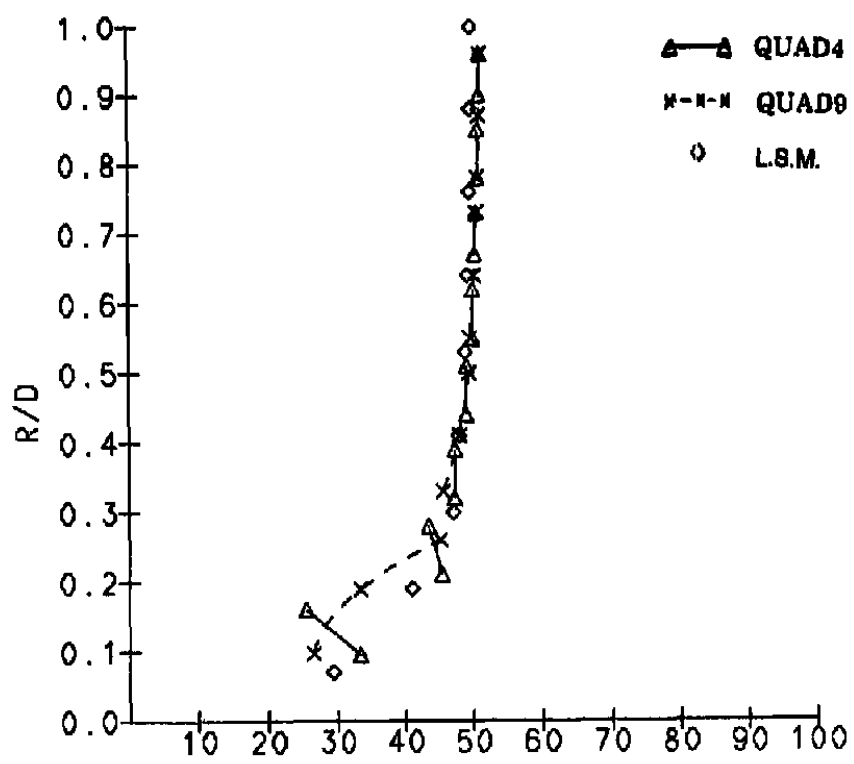

RADIAL STRESS

Fig. 6. Distribution $\sigma_{r r h}$ along a $45^{\circ}$ radial line for GL/EP $45^{\circ}$ and a type-III mesh ( $R$ is the distance from the hole center). 


\section{Mesh adaptation}

There are, typically, three methods of mesh adaptation: $h$-method; $p$-method; $r$-method. The first method increases the number of elements in regions in which interpolation errors are large. The second method increases the order of the polynomial approximation inside the elements with large interpolation errors. The third method relocates the nodes optimally by applying an optimality criterion, for a given number of elements and a prespecified order of polynomial approximation.

For the $h$-method case, the following problems are noted. A special refinement for transition from refined elements to coarse elements, or introduction of appropriate constraint conditions among degrees of freedom, is needed since not all meshes are subdivided. Now element connectivities must be reassigned. Thus much more computing time is required.

The comparisons performed between the QUAD4 and QUAD9 elements constitute a study of the $p$-method. The use of the QUAD9 gave only marginally better results, and so the $p$-method may not be advocated for anisotropic media. Here, the $r$-method with QUAD4 is chosen as the best procedure and it will now be described in greater detail.

The essence of the method is: given an initial, moderately refined grid, relocate the nodes to achieve

$$
\operatorname{Min}\left\{\operatorname{Max} E_{e}\right\}, \quad e=1,2, \ldots, \mathrm{NE},
$$

where $E_{e}$ is some appropriate element error measure. Diaz, Kikuchi and Taylor [8] treated the problem as one of optimization and showed that a necessary condition for optimality is $E_{\mathrm{c}}=$ constant, for all $e=1, \ldots, \mathrm{NE}$. Since the exact solution is unknown, computationally the optimality condition is approximated by

$$
E_{\mathrm{c}}^{h}=\text { constant for all } e=1, \ldots, \mathrm{NE} \text {. }
$$

Consider the error measure

$$
E_{e}=\left\{\int_{\Omega_{e}}\left(\sigma_{i j}-\sigma_{i j 1}\right)\left(\sigma_{i j}-\sigma_{i j \mathrm{I}}\right) \mathrm{d} \Omega_{e}\right\}^{1 / 2}
$$

with piecewise constant interpolation of the stresses.

A two-term Taylor expansion in $s$ and $t$ about the centroid of the master element for the stresses yields, where $s$ and $t$ are master element coordinates,

$$
\sigma_{i j}-\sigma_{i j \mathrm{I}}=\sigma_{i j}(s, t)-\sigma_{i j}(0,0)=s \frac{\partial \sigma_{i j}}{\partial s}+t \frac{\partial \sigma_{i j}}{\partial t}
$$

where $\sigma_{i j}(0,0)$ is the value of $\sigma_{i j}$ at the centroid, and the interpolated stress $\sigma_{i j \mathrm{I}}$ has the same value.

Then an approximate error measure using the finite element approximation of stressis, $\sigma_{i j h}$, can be written as

$$
E_{e}^{h}=\left\{\int_{-1}^{1} \int_{-1}^{1}\left(s \frac{\partial \sigma_{i j h}}{\partial s}+t \frac{\partial \sigma_{i j h}}{\partial t}\right)\left(s \frac{\partial \sigma_{i j h}}{\partial s}+t \frac{\partial \sigma_{i j h}}{\partial t}\right) J \mathrm{~d} s \mathrm{~d} t\right\}^{1 / 2},
$$

where $J$ is the determinant of the Jacobian matrix.

Note that $\sigma_{i j h}$ is not the stress

$$
\hat{\sigma}_{i j^{\prime}}=E_{i j k l} \varepsilon_{k l}\left(u_{h}\right)
$$


computed by using the constitutive equation and the finite element solution $u_{h}$. $\sigma_{y j h}$ is spanned by the same shape functions for the displacement $u_{h}$, i.e.,

$$
\sigma_{i j h}=\sum_{\alpha=1}^{4} \sigma_{i j \alpha} N_{\alpha}(s, t) \text { in } \Omega_{e},
$$

where $\sigma_{i j \alpha}$ are the values (unknown at this stage) of $\sigma_{i j h}$ at the four nodes in an arbitrary element $\Omega_{e}$. The stresses $\sigma_{i j \alpha}$ are computed from the least-squares procedure (a smoothing operation):

$$
\operatorname{Min}_{\sigma_{i j \alpha}} \frac{1}{2} \sum_{e=1}^{\mathrm{NE}} \int_{\Omega_{e}}\left(\sum_{\alpha=1}^{4} \sigma_{i j \alpha} N_{\alpha}(s, t)-\hat{\sigma}_{i j h}\right)^{2} \mathrm{~d} \Omega .
$$

Although $\hat{\sigma}_{i j h}$ is not continuous across the element boundaries, $\sigma_{i j h}$ is. Furthermore, $\sigma_{i j h}$ is differentiable in $s$ and $t$ in each element $\boldsymbol{\Omega}_{e}$.

$\partial \sigma_{i j h} / \partial s$ and $\partial \sigma_{i j h} / \partial t$ are approximated by their values at the centroid in each element. Then the error measure in (30) is approximated by

$$
E_{e}^{h}=\left\{\frac{4}{3} J\left[\left(\frac{\partial \sigma_{i j h}}{\partial s}\right)\left(\frac{\partial \sigma_{i j h}}{\partial s}\right)+\left(\frac{\partial \sigma_{i j h}}{\partial t}\right)\left(\frac{\partial \sigma_{i j h}}{\partial t}\right)\right]\right\}^{1 / 2} .
$$

In the plate with a hole, $\sigma_{\theta \theta}$ is the dominant stress component and an error measure was constructed using only this component in (34). The results were compared with those obtained using the full error measure and no appreciable differences were seen. In the sequel, the error measure involving $\sigma_{\theta \theta}$ alone is used for simplicity.

One further study of the error measure was performed for the QUAD4 element by taking a five-term Taylor expansion in $s$ and $t$ about the midpoints between the Gaussian integration points, as opposed to the centroid as used previously. The error measure based on this resulted in oscillations of meshes and no improvements.

The nodes must be relocated until the approximate condition $E_{e}^{h}=$ constant is satisfied. Here the scheme used by Diaz, Kikuchi and Taylor [8] is employed. The $n$th node is moved to the point $\left(x_{1}^{(n)}, x_{2}^{(n)}\right)$, where

$$
x_{i}^{(n)}=\left[\sum_{e=\alpha}^{\alpha+4} x_{i e}\left(E_{e}^{h} / A_{e}\right)\right] /\left(\sum_{e=\alpha}^{\alpha+4} E_{e}^{h} / A_{e}\right),
$$

where $\alpha$ is an element number, and $x_{i e}$ are the coordinates of the centroid of the eth element. Note that the summation is over the four elements neighboring the $n$th node.

This scheme implies that the new location of the nth nocie becomes closer to the centroid of the element which has the largest error measure among them. There is the possibility of 'nonconvexity', that is, the determinant of the Jacobian matrix in the normalized coordinate system may become nonpositive. Geometrically, this corresponds to an element with angles $>180^{\circ}$. Such elements are not allowed. If at any iteration step nonconvexity occurs, the relocation of the node responsible is skipped.

Another item of concern is the least-squares method, (33). The method computes continuous first derivatives (i.e., stresses) using the discontinuous first derivatives generated by the 
finite element solution. If small and large finite elements are arranged alternately, the method gives artificially high gradients in the small elements. Since the error measures are computed using derivatives of stresses, an artificially larger error is assigned to small elements. This nature can destroy convergence in the $r$-method. To avoid this misbehavior, one sets up a minimum area in the code.

Finally it should be noted that the node relocation scheme, (35), involves two aspects: diminution of error measures and smoothing of the meshes. These two aspects work in harmony for rectangular domains covered by nearly rectangular finite elements. However, they are almost contradictory close to the curved boundary, with its large curvatures. The relocation scheme tends to pass through the optimality condition in order to form very small elements. It has been observed that the maximum error measure first monotonically decreases and then starts to increase. Thus it is recommended that one monitors the value of the maximum error and that the adaptive prosess be terminated when it starts increasing. This feature is one of the drawbacks of the relocation scheme, (35).

\section{Computational procedure for shape optimization}

The optimality condition postulated previously requires that the values of the failure function be constant along the design boundary. The shape of the design boundary represented by the nodes should be moved to satisfy the optimality condition and the area constraint. A method of node moving following Chung [20] is adopted.

Suppose there are $N$ nodes on the design boundary $\Gamma_{\mathrm{d}}$. To achieve optimality, these are moved, in an iterative fashion, to new locations by means of

$$
x_{i}^{k+1}=x_{i}^{k}+\Delta x_{i}^{k}, \quad i=1,2, \ldots, N,
$$

where $x$ denotes position vector and $k$ is an iteration index. The vector $\Delta x_{i}^{k}$ has a magnitude $\delta_{i}^{k}$ and a direction $\boldsymbol{\alpha}_{i}^{k}$ :

$$
\Delta x_{i}^{k}=\delta_{i}^{k} \alpha_{i}^{k}
$$

The overall scheme is outlined in Fig. 7. There NDE and NOP denote nodes on the design boundary and on the corresponding (by means of the vector $\alpha_{i}^{k}$ to be described) opposite outer boundary. $s_{i}$ is the arc length between two neighboring boundary segments. The choice of $\delta_{1}^{k}$ and $\boldsymbol{\alpha}_{1}^{k}$ will now be discussed in more detail. The magnitude factor is taken to be

$$
\delta_{i}^{k}=\frac{\Phi_{i}-\beta}{\Phi_{\max }-\beta} \delta_{\max }^{k},
$$

where $\Phi_{1}$ is the value of the failure function at $i$ th node, $\beta$ is the average value of $\Phi$ over the nodes, i.e.,

$$
\beta=\frac{1}{N} \sum_{i=1}^{N} \Phi_{1}
$$




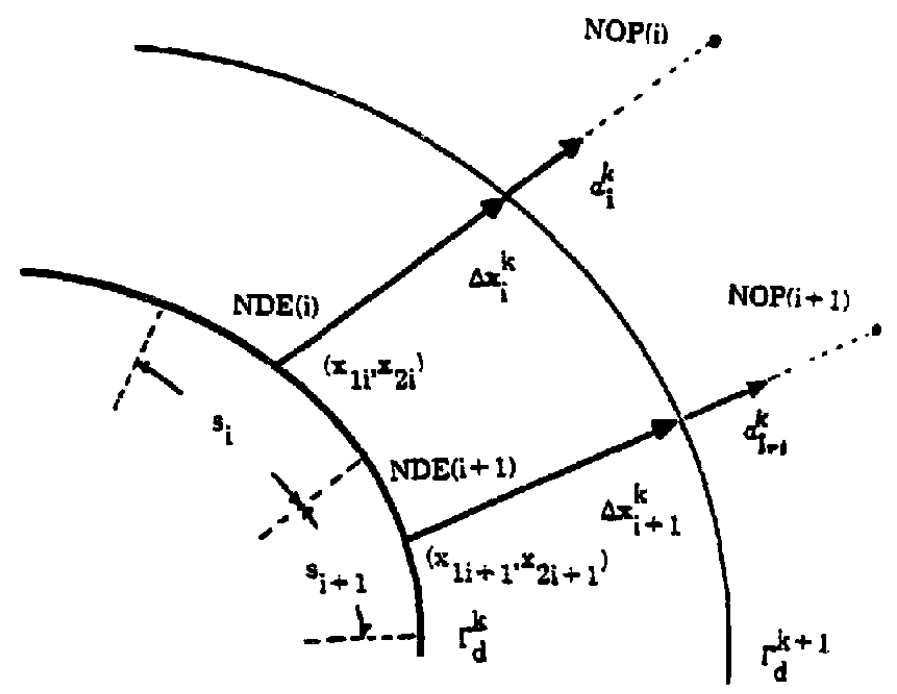

Fig. 7. Design node relocation scheme.

An initial moving constant $\delta_{\max }^{1}$ is assigned and the value of $\delta_{\max }^{k}$ in the successive iterations is determined from

$$
\delta_{\max }^{k}=\delta_{\max }^{1}-\left(\frac{\delta_{\max }^{1}-\bar{\delta}}{\mathrm{NI}-1}\right)(k-1),
$$

where NI denotes the number of anticipated iterations (an assigned value). The parameter $\bar{\delta}$ is usually taken as $1 \%$ of $\delta_{\max }^{1}$. The value of $\delta_{\max }^{1}$ dictates rate of convergence. If the value of $\delta_{\text {max }}^{1}$ is big, the convergence speed is fast but is sometimes accompanied by oscillations of the design boundary. The range of $\delta_{\max }^{1}$ is taken to be

$$
0<\delta_{\max }^{1}<s_{\text {ave }}^{1}
$$

where $s_{\text {ave }}^{1}$ denotes the average value of $s_{i}$ for the first iteration. The value of $\delta_{\max }^{1}$ is taken as $25 \%$ of $s_{\text {ave }}^{1}$. This was found from many numerical experiments to be a good choice.

The direction vector $\alpha_{i}^{k}$ is taken to be the normal vector calculated as follows:

$$
\alpha_{i}^{k}=\frac{x^{k}(\mathrm{NDE}(i))-x^{k}(\mathrm{NOP}(i))}{\left|x^{k}(\mathrm{NDE}(i))-x^{k}(\mathrm{NOP}(i))\right|}
$$

After the new coordinates of the design nodes are obtained, the interior nodes between the design nodes and the opposite outer boundary nodes should also be moved, in a proportional fashion, to keep a proper mesh (e.g., "no crashing"). This is done as follows:

$$
\Delta x^{k}(\mathrm{NIN}(i))=\Delta x_{i}^{k} \frac{\left|x^{k}(\mathrm{NIN}(i))-x^{k}(\mathrm{NOP}(i))\right|}{\left|x^{k}(\mathrm{NDE}(i))-x^{k}(\mathrm{NOP}(i))\right|}
$$

where NIN $(i)$ denotes the interior nodes assoriated with the $i$ th design node. 
The iteration is stopped through use of the quantity

$$
\mathrm{DTP}=\left\{\frac{1}{N} \sum_{i=1}^{N}\left(s_{i}^{k} \frac{\Phi_{1}-\beta}{\beta}\right)^{2}\right\}^{1 / 2} \frac{1}{s_{\mathrm{ave}}^{k}},
$$

where $s_{\text {ave }}^{k}$ is the average value of $s_{i}^{k}$. When DTP $\leqslant 0.01$, the iteration process is terminated.

The overall procedure was checked as follows. A glass/epoxy $45^{\circ}$ plate, with a circular hole, under a uniaxial tension of $100 \mathrm{~N} / \mathrm{cm}^{2}$ was treated, using both uniformly refined meshing and the $r$-method. It should be pointed out that the $r$-method will not be good unless original mesh is reasonably accurate [26]. Results on $\sigma_{\theta \theta}$ are shown in Table 2 and Fig. 8. In Fig. 8, the solid line and the dotted line represent Lekhnitskii's solution (equation (25)) and the finite element

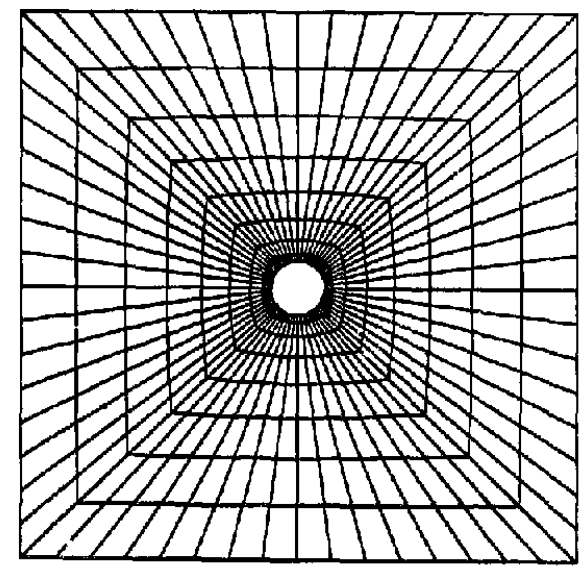

Orig, mesh

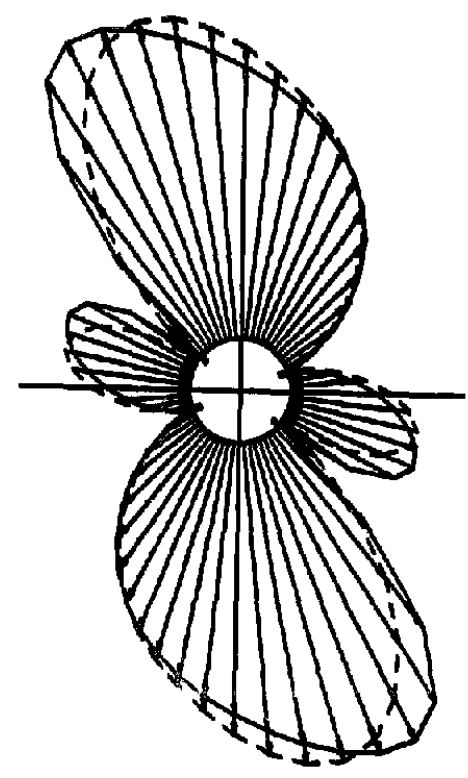

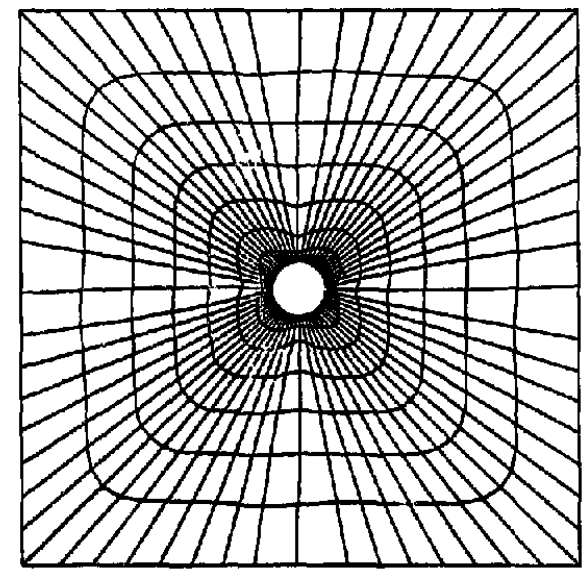

$r \cdot$ mesh

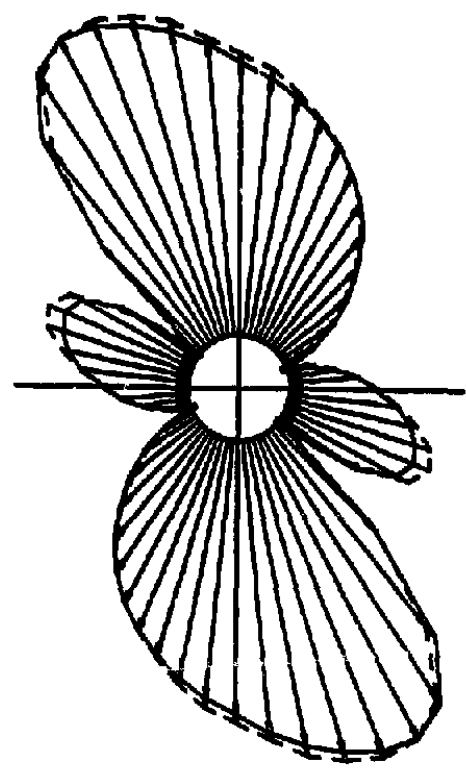

Distribution of ${ }_{B \theta}$ Around the Hole

Fig. 8. Application of the $r$-method to a ply, with a circular hole, under uniform tension. 
Table 2

Comparison of Max $\left|\sigma_{\theta \theta}-\sigma_{9 \theta h}\right|$ with / without $r$-meshes (unit: $100 \mathrm{~N} / \mathrm{cm}^{2}$ )

\begin{tabular}{rcc}
\hline & \multicolumn{2}{c}{ QVAD4 } \\
\cline { 2 - 3 } Mesh type & Original mesh & r-mesi \\
\hline I $(\mathrm{NX}=160)$ & 140.00 & 51.16 \\
II $(\mathrm{NX}=336)$ & 92.81 & 32.41 \\
III (NX = 576) & 65.25 & 17.17 \\
& $(20.0 \%)$ & $(5.2 \%)$ \\
\hline
\end{tabular}

Table 3

Comparison of Max $\sigma_{\theta \theta h}$ (unit $100 \mathrm{~N} / \mathrm{cm}^{2}$ )

\begin{tabular}{rccccc}
\hline & \multicolumn{2}{c}{ Isotropic } & & \multicolumn{2}{c}{ Composite (GL/EP 45 ) } \\
\cline { 2 - 3 } \cline { 6 - 6 } Mesh type & $\max \sigma_{\theta \theta h}$ & DP (\%) & & $\max \sigma_{\theta \theta h}$ & DP (\%) \\
\hline I $(\mathrm{NX}=160)$ & 246.80 & - & & 209.80 & - \\
II $(\mathrm{NX}=336)$ & 288.40 & 16.9 & & 265.80 & 26.7 \\
III $(\mathrm{NX}=576)$ & 301.90 & 4.7 & & 276.90 & 4.2 \\
\hline
\end{tabular}

solution, respectively. Noticeable improvement in the accuracy is seen with almost $15 \%$ error reduction.

In general an analytic solution is not available for comparison. In these cases' a useful operational procedure can be inferred from the data. Table 3 shows a comparison of the maximum value of $\sigma_{\theta \theta h}$. In the table, DP denotes $\left(\max \left|\sigma_{\theta \theta h}^{i+1}-\sigma_{\theta \theta h}^{i}\right|\right) /\left(\max \sigma_{\theta \theta h}^{i}\right)$, where $\max \sigma_{\theta \theta h}^{i}$ denotes the value of $\max \sigma_{\theta \theta h}$ using the $i$ th-type mesh. The change of the maximum value of $\sigma_{\theta \theta h}$ using the type-III mesh was $4.7 \%$ compared to the maximum value of $\sigma_{\theta \theta h}$ using the type-II mesh. This corresponds to $20 \%$ error between the finite element solution and the analytic solution using the type-III mesh for the case with known analytic solution. Based on this experience, it is suggested that the $r$-method be used when the change of the maximum value of $\sigma_{\theta \theta h}$ in an iteration is within around the $5 \%$ range. This strategy will be adapted in later sections.

\section{Validation. Isotropic plate with a hole}

For isotropic media, the failure function $\Phi$ is the Von Mises stress. It has been shown analytically that for an infinite isotropic plate under biaxial loading the optimal hole shape is an ellipse with a semiaxis ratio the same as the stress components at infinity (see [6]). The isotropic case is examined here as a check on the procedures and the computer program. Biaxial loading with a ratio of $\sigma_{1} / \sigma_{2}=1.5$ is applied. The initial hole shape is taken to be circular, with the dimensions of the plate and the material properties the same as in Section 5.

The strategy discussed earlier is used. The change in the maximum value of $\sigma_{\theta \theta h}$ is examined for QUAD4 meshes to find out an appropriate number of elements for which the $r$-method should be applied. A scheme with $\mathrm{NE}=\mathbf{5 1 2}$ (type-III mesh) was chosen, since the change in going from type-II to type-III mesh was only $3.23 \%$. The $r$-method is applied to this initial 

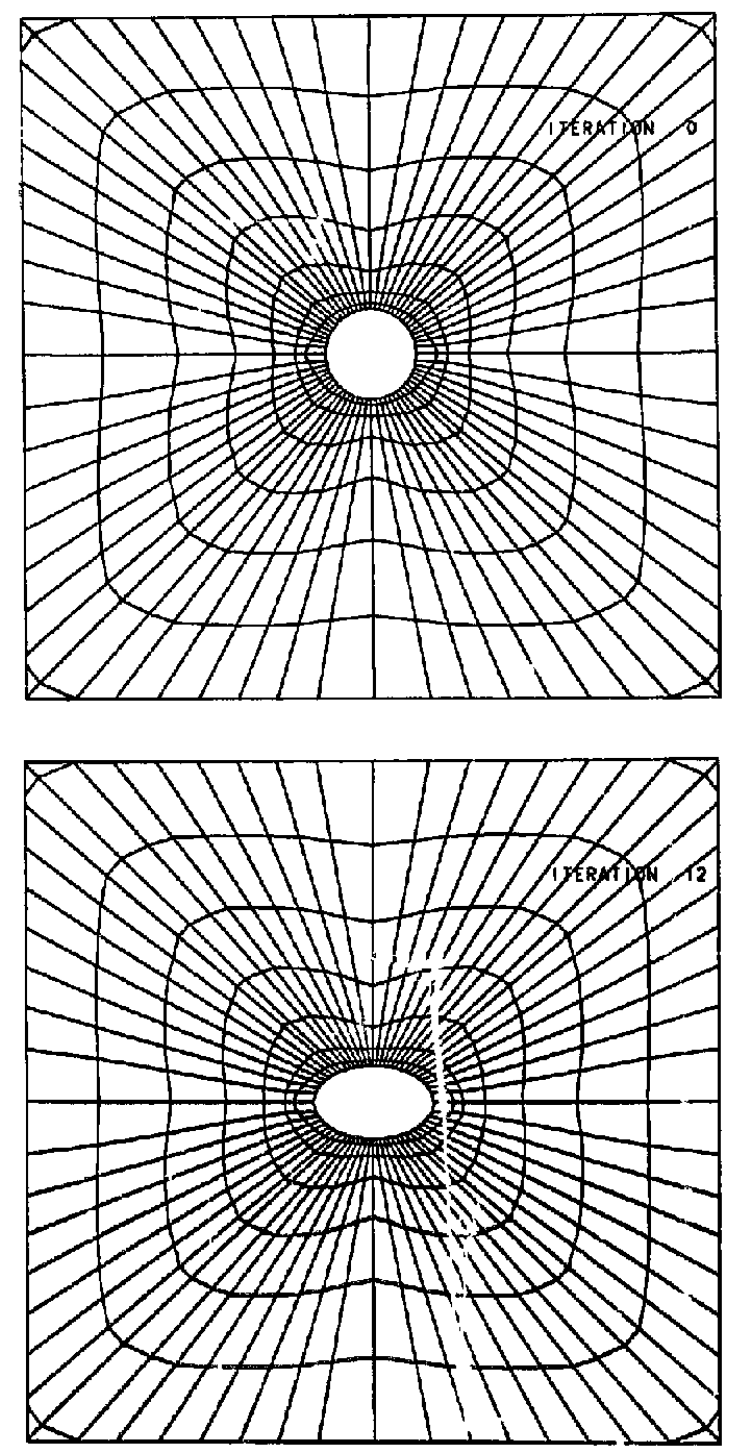

Fig. 9. The initial and final shapes for an isotropic plate.

mesh and then the shape optimization procedure is used. No further use of the $r$-method after the initial application was found necessary to obtain good results.

The initial and final shapes are shown in Fig. 9. Convergence was achieved with 12 iterations. The final hole shape is an ellipse with a semiaxis ratio of 1.55 , which differs only by $3.3 \%$ from the analytic result.

\section{Single lamina with a hole}

In this section a single lamina with a $45^{\circ}$ fiber angle is studied to find out the optimal hole shape under biaxial loading. The biaxial loads are applied with a ratio of 1 . The plate material is glass-epoxy. with properties as given in Table 1. Using the criteria described before, a type-III type mesh $(\mathrm{NELX}=512)$ was chosen as the initial mesh. 
An interesting issue is whether the $r$-method should be just applied initially, or applied several times as the node relocation scheme to achieve optimization progresses. A study was done on this issue, with Tsai-Hill failure chosen as the criterion.

The $r$-method was applied after each design iteration. This was not successful. Oscillating meshes occurred, with the process terminating after 9 iterations. The reason for the failure can be traced to the nature of the least-square method, as discussed in Section 6 . The shape optimization scheme is generating alternate large and small elements near the boundary and is leading to unacceptable errors in the $r$-method. This approach was abandoned.

Figure 10 shows the initial and final shapes, with the $r$-method applied at the initial stage only. Figure 11 shows the final shape with the $r$-method applied also at the fifth and tenth iterations. Note that the final shapes are practically identical. The conclusion drawn is that even though there was some reduction in the number of iterations required, an initial
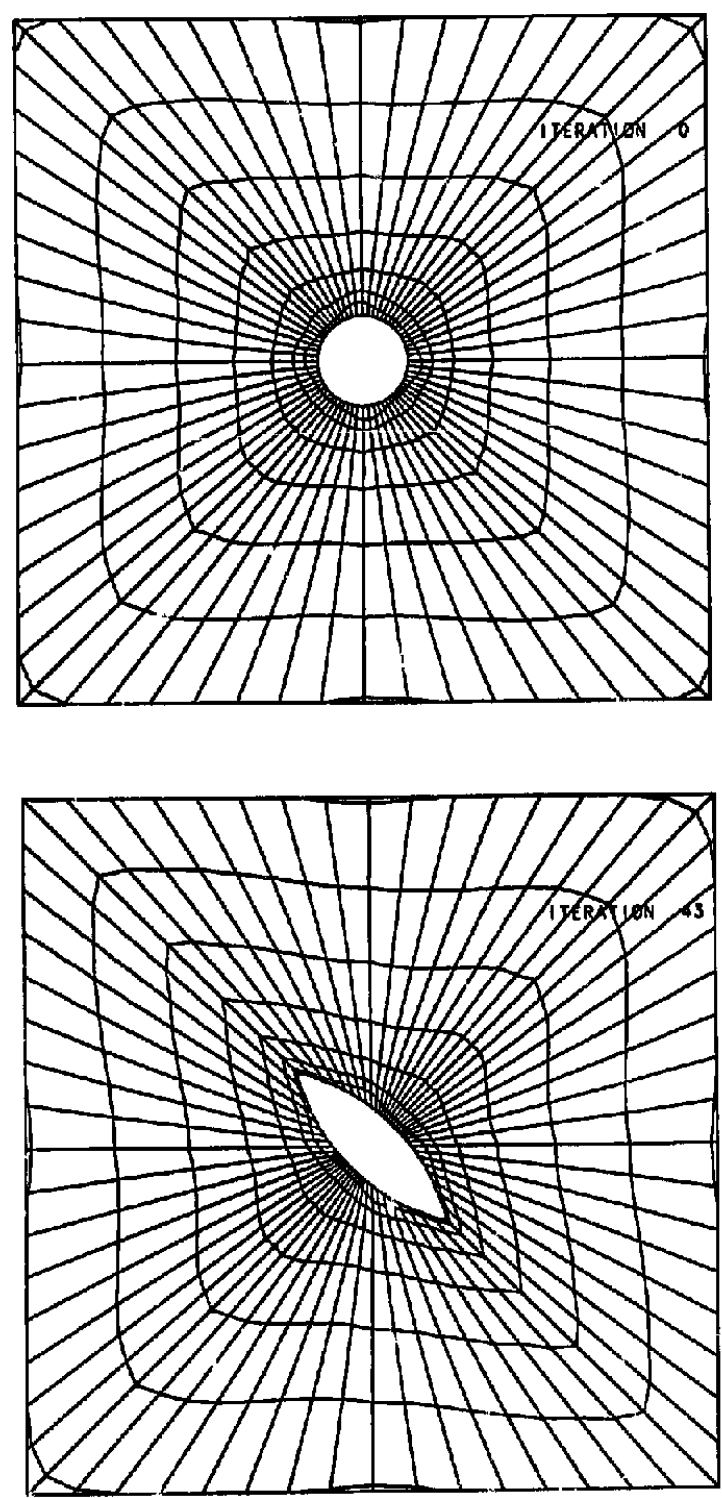

iig. 10. The initial and final shapes for a GL/EP $45^{\circ}$ plate using the Tsai-Hill failure function. 


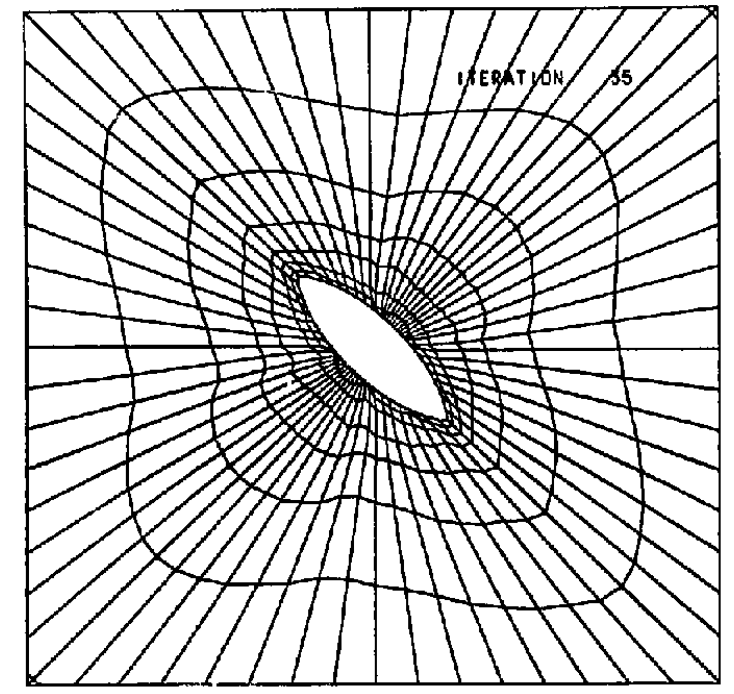

Fig. 11. The final shape for a GL/EP $45^{\circ}$ plate with the $r$-method applied after several shape optimization iterations.

$r$-method is all that is required in the current work. Further discussion on results will now be given.

Figure 12 shows the final shape using the Tsai-Wu criterion. An elliptical shape is again seen, with a semiaxis ratio of 3.8 (compared to 3.5 using Tsai-Hill, Fig. 10).

Figure 13 shows the distribution around the hole boundary of the failure functions for both the initial and final shapes. It is seen that the postulated optimality condition of $\Phi=$ constant is achieved exactly. The maximum value, and the difference between the average and the minimum, of the Tsai-Wu failure function are always larger than those of Tsai-Hill. This induces sharper corners in the optimal shape when Tsai-Wu failure is used instead of Tsai-Hill failure.

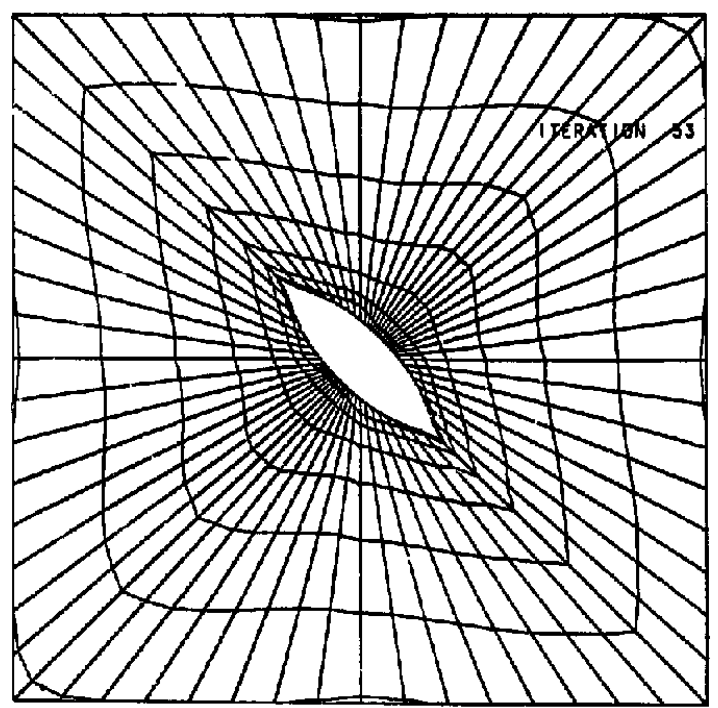

Fig. 12. The final shape for a GL/EP $45^{\circ}$ plate using the Tsai-Wu failure function. 

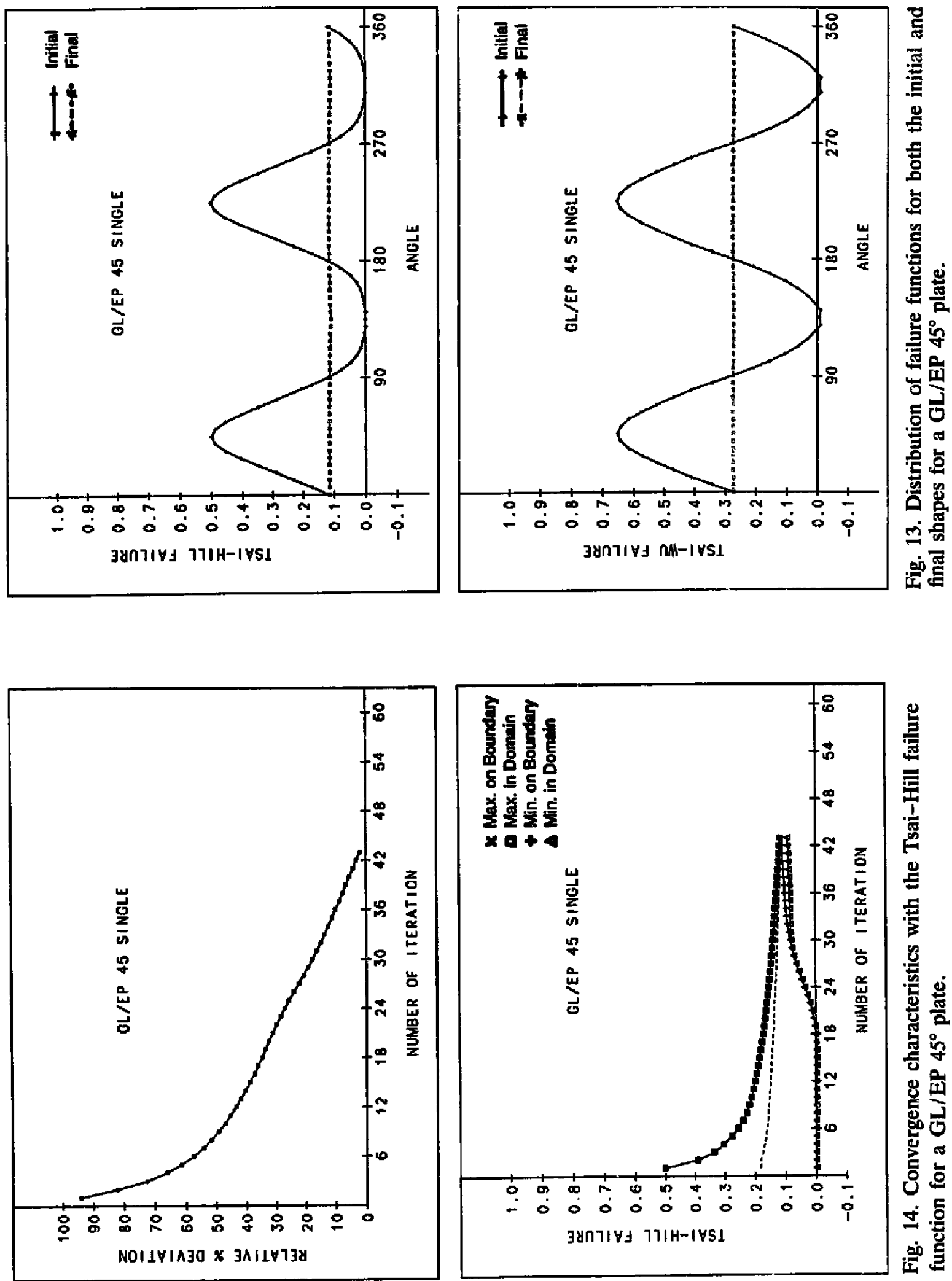

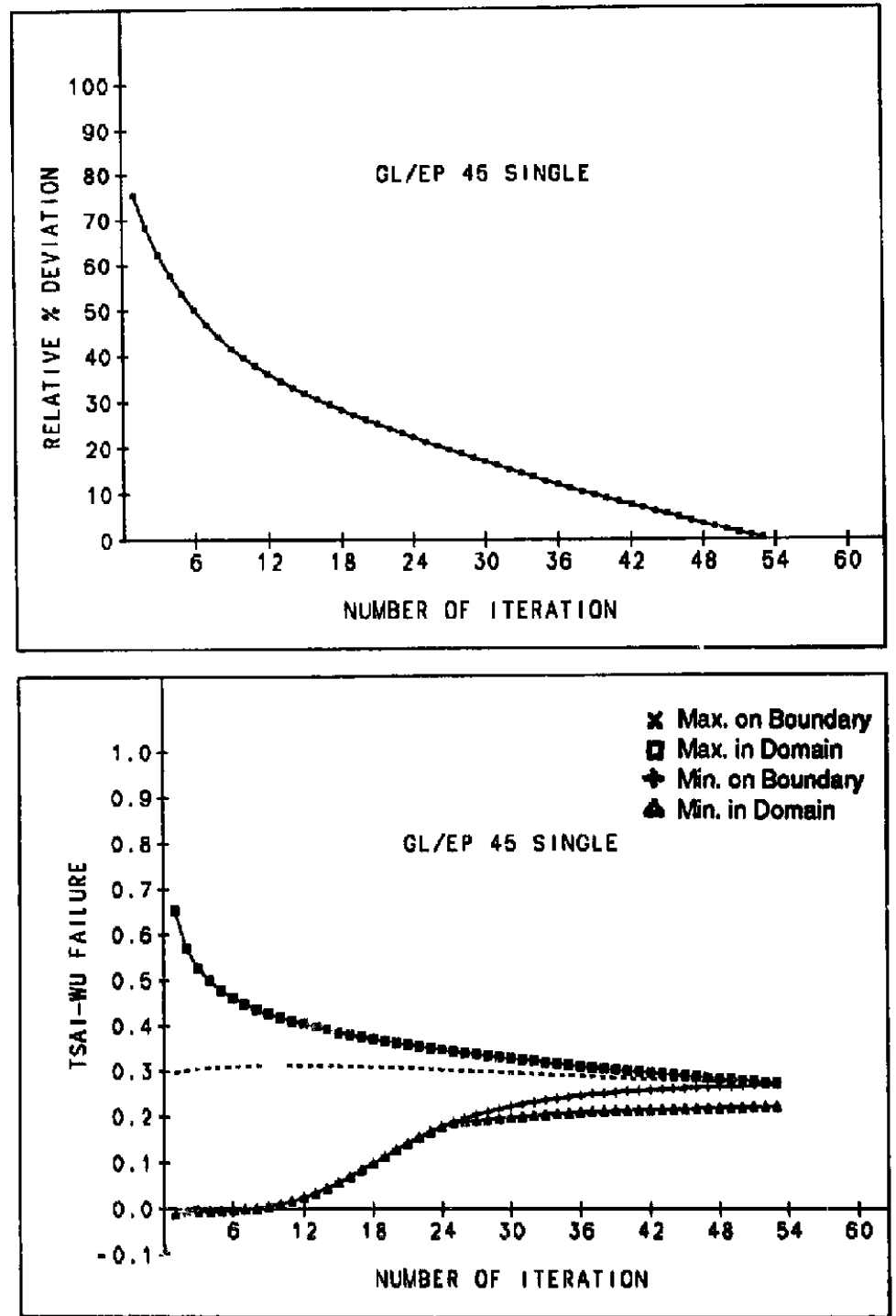

Fig. 15. Convergence characteristics with the Tsai-Wu failure function for a GL/EP $45^{\circ}$ plate.

Convergence characteristics are shown in Figs. 14 and 15 (relative \% deviation is DTP as defined by (42)). Note that the number of iterations is about five times bigger than the number for the isotropic case.

\section{Multilayered laminates with a hole}

All the laminates treated here are symmetric and are made of glass-epoxy plies with properties as given in Table 1. Biaxial loads in a one-to-one ratio are applied. Optimization is based on using average failure functions, as discussed in connection with (24).

Treated first is a two-ply with iay up $\left[0,9^{\circ}\right]_{s}$. The initial and final shapes for both Tsai-Hill and Tsai-Wu failure are shown in Figs. 16 and 17. Now, instead of ellipses, the optimum shapes are more like squares with rounded shoulders. Both criteria lead to very similar shapes 

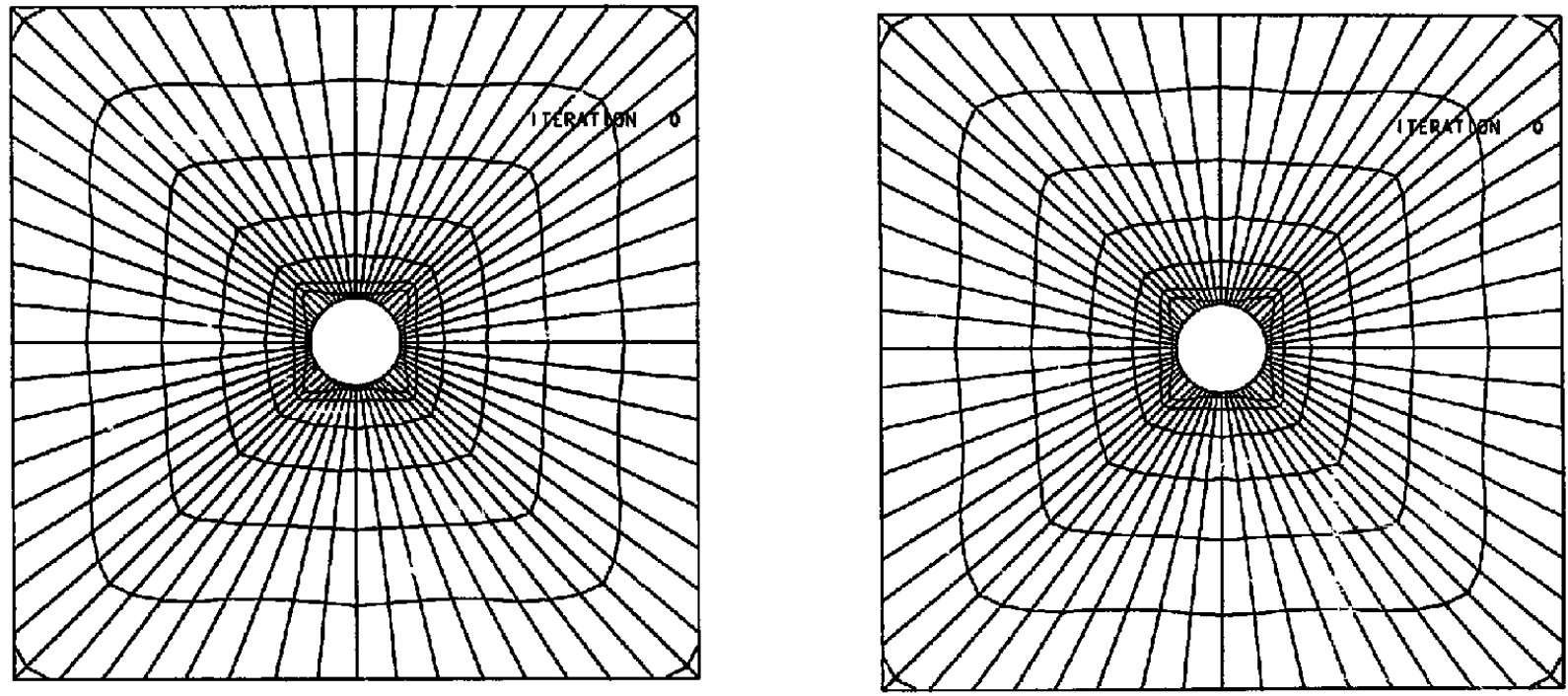

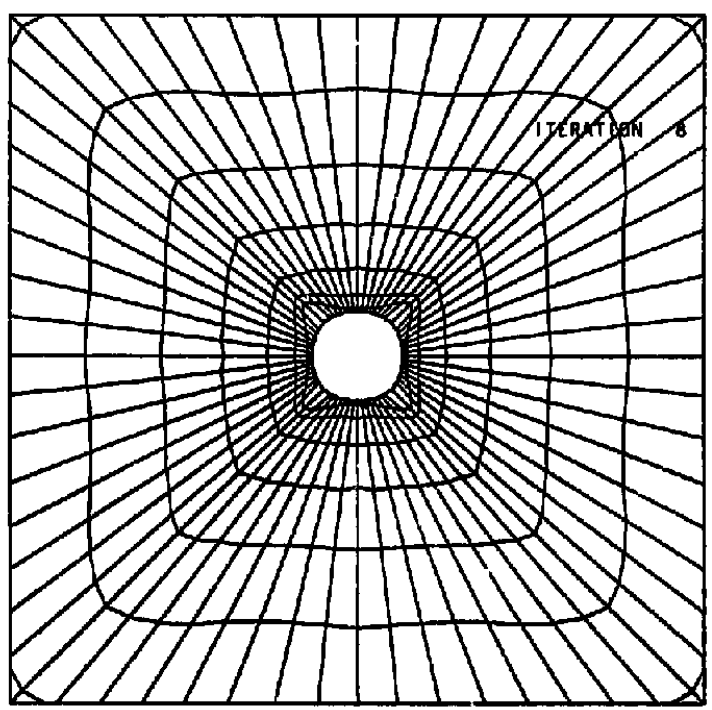

Fig. 16. The initial and final shapes for a GL/EP $[0,90]_{s}$ plate using the Tsai-Hill failure function.

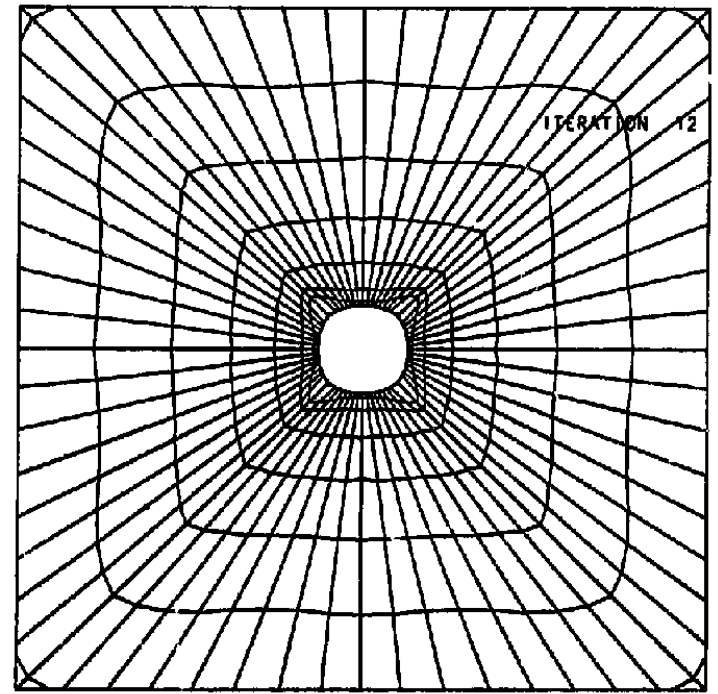

Fig. 17. The initial and final shapes for a GL/EP $[0,90]_{s}$ plate using the Tsai-Wu failure function.

(true also in the sequel, for which only results based on Tsai-Hill are presented). Figure 18 gives the distribution of the failure function around the hole boundary. Note that the postulated optimality condition is achieved precisely. Convergence was achieved in about twelve iterations, much quicker than for the single lamina, due, apparently, to the smoothing involved in the averaging process.

The next case studied is a three-ply with lay up $\left[0^{\circ} / \pm 45^{\circ}\right]_{s}$. The initial and final shapes are shown in Fig. 19. An elliptical final shape is now observed, with a major axis in the direction transverse to the $0^{\circ}$ fiber. The ratio of major to minor axis is 1.63 (it is 1.81, using Tsai-Wu). It was found that the optimality condition was again achieved precisely.

Further cases can be found in [26], involving other lay-ups and more plies. Results similar to the ones discussed above were found. 


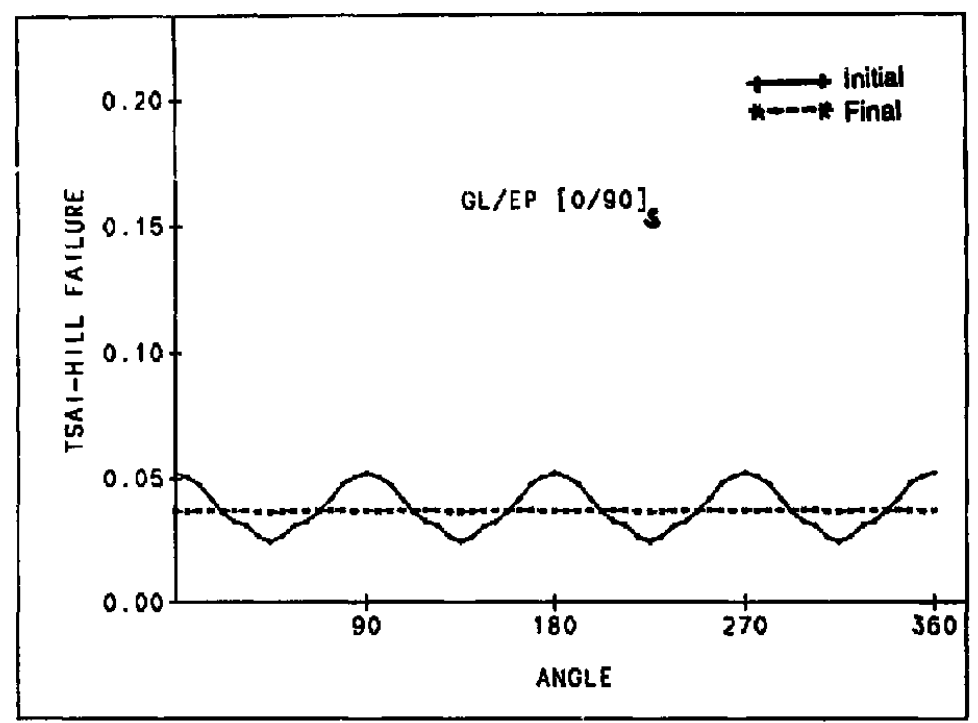

Fig. 18. Distribution of Tsai-Hill failure function for both the initial and final shapes in a GL'EP $[0,90]_{s}$ plate.
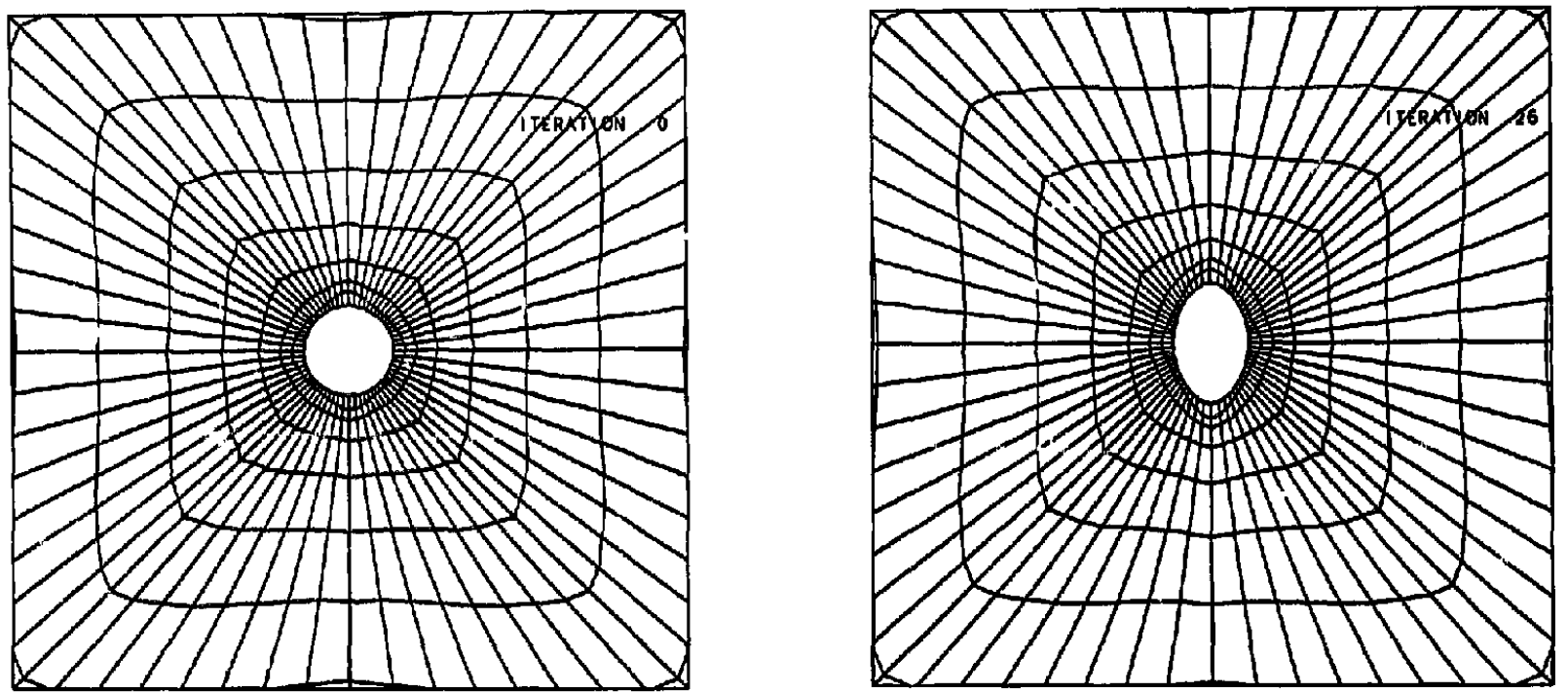

Fig. 19. The initial and final shapes for GL/EP $[0 / \pm 45]_{s}$ plate using the Tsai-Hill failure function.

\section{Isotropic plate with a pin-loaded hole}

A nat"ral extension of the work reported here is shape optimization of pin-loaded holes. Here a preliminary pilot study is presented, using the postulated optimality condition and a postulated stress distribution at the pin boundary. The Von Mises failure function is used.

A plate with a pin-loaded hole is shown in Fig. 20. The pin is taken to be rigid and fixed in space. Friction is neglected and $180^{\circ}$ contact is assumed. For equilibrium one must have

$$
\frac{D}{2} \int_{-\pi / 2}^{\pi / 2} q \cos \theta \mathrm{d} \theta=p
$$

where $p$ is the total applied load, and $q$ is the normal traction on the hole boundary. Here $q$ is 


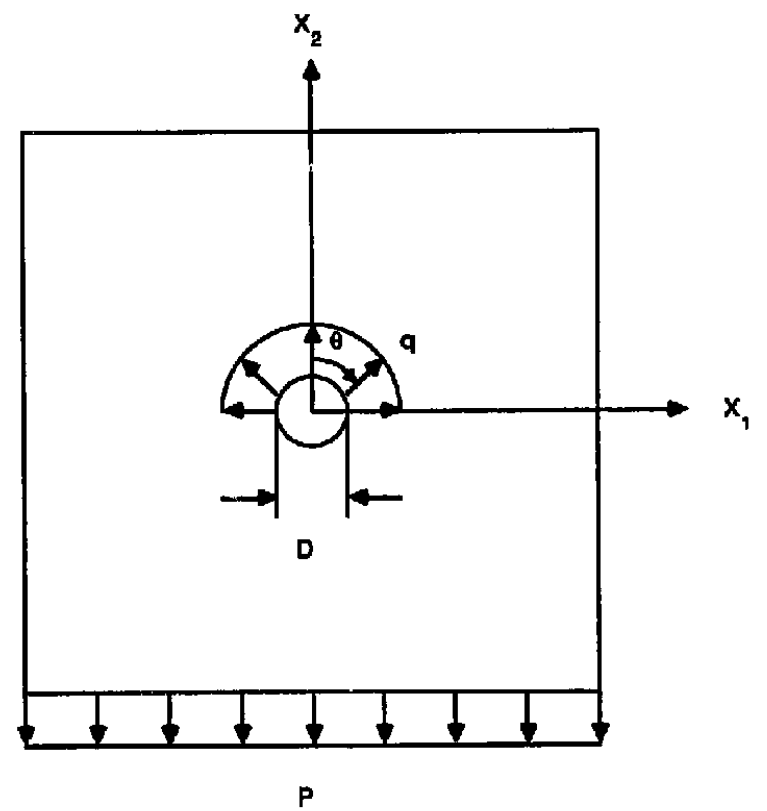

Fig. 20. A plate with a pin-loaded hole.
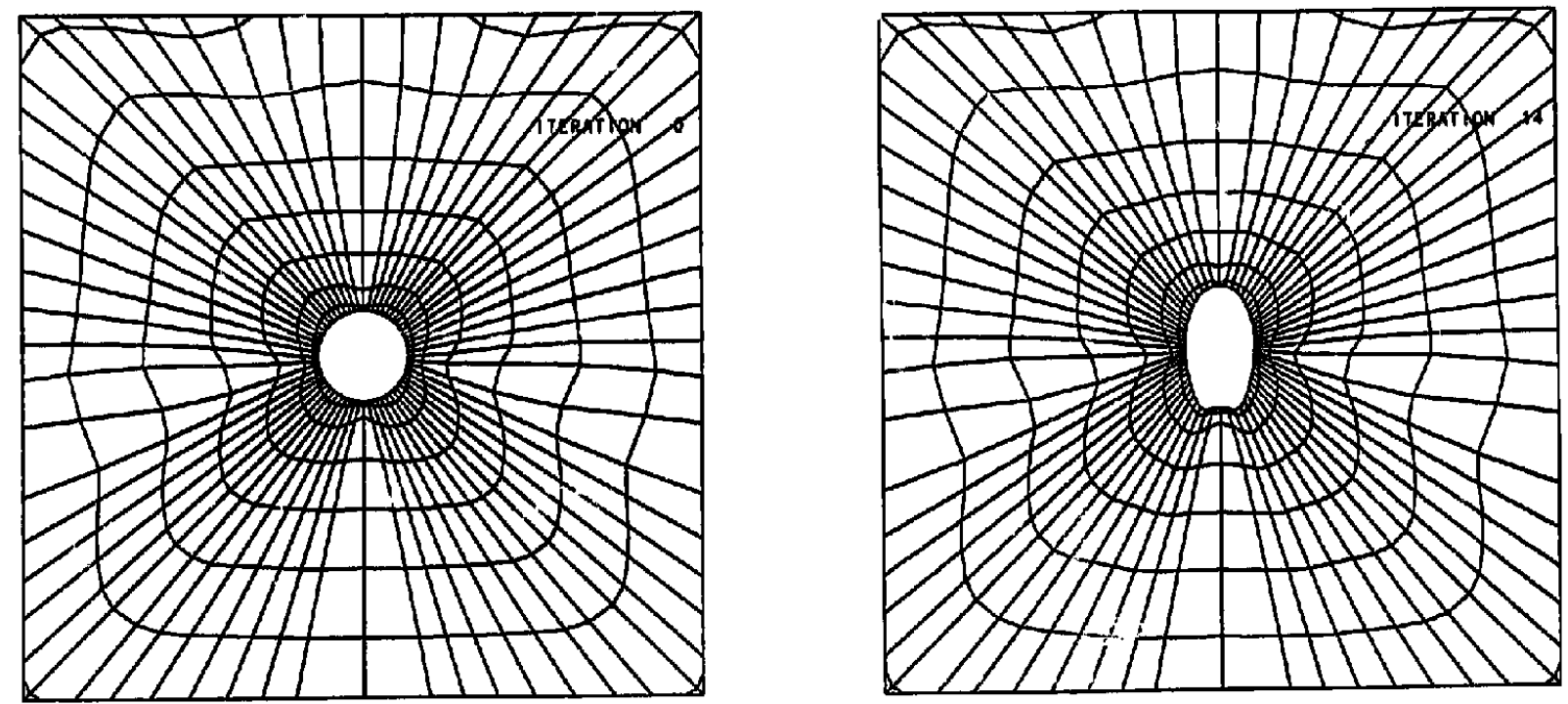

Fig. 21. The initial and final shapes for isotropic plate with a pin-loaded hole.

assumed uniform. As the pin and hole shapes change, $q$ also changes and must be updated according to (43).

The initial and final shapes are shown in Fig. 21. An oval-like final shape is seen. Now, however, final convergence was not achieved. The distribution of the Von Mises stress along the hole boundary is shown in Fig. 22. The optimality condition is not fully achieved. However the final shape involves a $25 \%$ reduction in peak stress and the design could be regarded as better. Whether the manufacturing difficulties involved in such shapes are worth $25 \%$ stress drops must be considered a debatable point at this stage. 


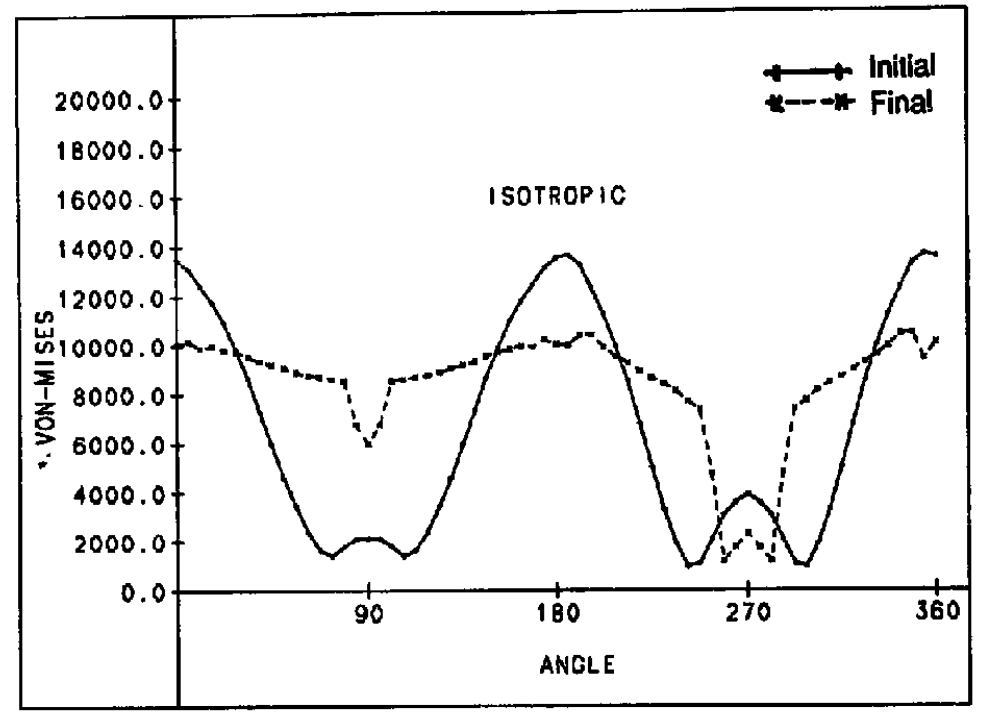

Fig. 22. Distribution of Von Mises stress for both the initial and final shapes in an isotropic plate with a pin-loaded hole.

\section{Note}

This paper was submitted in 1987. In the review process, the related work of Bäcklund and Isby [28] has been brought to our attention.

\section{References}

[1] E.J. Haug, A unified theory of optimization of structures with displacements and compliance constraints, J. Structural Mech. 9 (1981) 415-437.

[2] R.T. Haftka and R.V. Grandhi, Structural shape optimization-a survey, Comput. Meths. Appl. Mech. Engrg. 57 (1986) 91-106.

[3] K.K. Choi and E.J. Haug, Shape design sensitivity analysis of elastic structures, J. Structural Mech. 11 (1983) 231-269.

[4] K. Dems and Z. Mroz, Variational approach by means of adjoint systems to structural optimization and sensitivity analysis-I, Internat. J. Solids and Structures 19 (1983) 677-692.

[5] N.V. Banichuck, Optimality conditions in the problem of seeking the hole shapes in elastic bodies, Appl. Math. Mech. 41 (1977) 920-925.

[6] N.V. Banichuck, Problems and Methods of Optimal Structural Design (Plenum Press, New York, 1983).

[7] N. Kikuchi, K.Y. Chung, T. Torigaki and J.E. Taylor, Adaptive finite element methods for shape optimization of linearly elastic structures, Comput. Meths. Appl. Mech. Engrg. 57 (1986) 67-89.

[8] A.R. Diaz, N. Kikuchi and J.E. Taylor, A method of grid optimization for finite element methods, Comput. Meths. Appl. Mech. Engrg. 45 (1983) 29-45.

[9] V. Braibant and C. Fleury, Shape optimal design using B-splines, Comput. Meths. Appl. Mech. Engrg. 44 (1984) 247-267.

[10] V. Braibant and C. Fleury, An approximation-concepts approach to shape optimal design, Comput. Meths. Appl. Mech. Engrg. 53 (1985) 119-148.

[11] T.P. Kicher and T.L. Chao, Minimum weight design of stiffened fiber composite cylinders, J. Aircraft 8 (1971) $562-568$.

[12] Y. Hi ano, Optimum design of laminated plates under axial compression, AIAA J. 17 (1979) 1017-1019. 
[13] Y. Hirano, Optimum design of laminated plates under shear, J. Composite Mat. 13 (1979) 329-334.

[14] W.J. Park, An optimal design of simple symmetric laminates under the first ply failure criterion, J. Composite Mat. 16 (1982) 341-355.

[15] T.R. Tauchert and S. Adibhatla, Design of laminated plates for maximum stiffness, J. Composite Mat. 18 (1984) 58-69.

[16] S.W. Tsai and H.T. Hahn, Introduction to Composite Materials (Technomic, Westport, CT, 1980).

[17] C. Yiping, Optimal design of a laminate containing an elliptical hole, Mech. Res. Comm. 11 (1984) 329-336.

[18] R.M. Jones, Mechanics of Composite Materials (Scripta Book Company, Washington, DC, 1975).

[19] O.A. Bauchau, Optimal design of high speed rotating graphite/epoxy shafts, J. Composite Mat. 17 (1983) 171-180.

[20] K.Y. Chung, Shape optimization and free boundary problems with grid adaptation, Ph.D. Dissertation, University of Michigan, Ann Arbor, MI, 1986.

[21] S.R. Soni, Tailure analysis of composite laminates with a fastener hole, ASTM STP 593 (1981) 145-164.

[22] T. Krauthammer, Accuracy of finite element method near a curved boundary, Comput. \& Structures 10 (1979) 921-929.

[23] R.J. Nuismer and J.M. Whitney, Uniaxial failure of composite laminates containing stress concentrations, ASTM STP 593 (1975) 117-142.

[24] S.G. Lekhnitskii, Anisotropic Plates (Gordon and Breach, New York, 1960).

[25] M. Hyer, Private Communication.

[26] N. Kikuchi, Finite Element Methods in Mechanics (Cambridge University Press, New York, 1986).

[27] M.S. Lee, Shape optimization of a hole in symmetric laminates, Ph.D. Dissertation, University of Michigan, Ann Arbor, MI, 1987.

[28] J. Bäcklund and R. Isby, Shape optimization of holes in composite shear panels, in: Proceedings IUTAM Symposium on Structural Optimization, Melbourne, Australia, 1988. 